\title{
Identity-Based Encryption with (Almost) Tight Security in the Multi-instance, Multi-ciphertext Setting
}

\author{
Dennis Hofheinz ${ }^{(凶)}$, Jessica Koch, and Christoph Striecks \\ KIT, Karlsruhe, Germany \\ \{Dennis.Hofheinz, Jessica.Koch, Christoph.Striecks\}@kit.edu
}

\begin{abstract}
We construct an identity-based encryption (IBE) scheme that is tightly secure in a very strong sense. Specifically, we consider a setting with many instances of the scheme and many encryptions per instance. In this setting, we reduce the security of our scheme to a variant of a simple assumption used for a similar purpose by Chen and Wee (Crypto 2013). The security loss of our reduction is $\mathbf{O}(k)$ (where $k$ is the security parameter). Our scheme is the first IBE scheme to achieve this strong flavor of tightness under a simple assumption.

Technically, our scheme is a variation of the IBE scheme by Chen and Wee. However, in order to "lift" their results to the multi-instance, multi-ciphertext case, we need to develop new ideas. In particular, while we build on (and extend) their high-level proof strategy, we deviate significantly in the low-level proof steps.
\end{abstract}

\section{Introduction}

Tight Security. For many cryptographic primitives, we currently cannot prove security directly. Hence, we typically reduce the security of a given scheme to the hardness of a computational problem, in the sense that every successful attack on the scheme yields a successful problem solver. Now it is both a theoretically and practically interesting question to look at the loss of such a reduction. Informally, the loss of a reduction quantifies the difference between the success of a hypothetical attacker on the cryptographic scheme, and the success of the derived problem solver. From a theoretical perspective, for instance, the loss of a reduction can also be viewed as a quantitative measure of (an upper bound for) the "distance" between primitive and assumption. But "tight" (or, "loss-free") reductions are also desirable from a practical perspective: the tighter a reduction, the better are the security guarantees we can give for a specific instance of the scheme. Hence, we can recommend smaller keylengths (which lead to more efficiency) for schemes with tighter security reduction.

However, in most practical usage scenarios, a cryptographic primitive is used multiple times. (For instance, in a typical multi-user encryption scenario,

D. Hofheinz - was supported by DFG grants GZ HO 4534/2-2 and GZ HO 4534/4-1.

J. Koch-was supported by BMBF project "KASTEL".

C. Striecks - was supported by DFG grant GZ HO 4534/2-2.

(c) International Association for Cryptologic Research 2015

J. Katz (Ed.): PKC 2015, LNCS 9020, pp. 799-822, 2015.

DOI: $10.1007 / 978-3-662-46447-2 \_36$ 
many instances of the encryption scheme are used to produce even more ciphertexts.) Hence, tight security reductions become particularly meaningful when they reduce an attacker on the whole system (with many instances of the cryptographic scheme) to a problem solver. In fact, while for many primitives (such as secret-key [2] or public-key [3] encryption), one-instance security is known to imply multi-instance security, the corresponding security guarantees for concrete schemes may indeed vanish in the number of instances [2].

Existing Tightly Secure Schemes. The loss of security reductions has been considered explicitly by Bellare et al. [2] for the case of encryption schemes. The first "somewhat tight" reductions (whose loss is independent of the number of instances of the scheme, but not of the number of ciphertexts) for public-key encryption (PKE) schemes could be given in [4]. In the following years, more tight (or somewhat tight) reductions for encryption schemes were constructed in the random oracle model $[7,10,14]$, or from " $q$-type" assumptions $[15,16] .{ }^{1}$

However, only recently, the first PKE schemes emerged $[1,18,20]$ whose tight security (in the multi-instance, multi-ciphertext setting) can be proved under simple assumptions in the standard model. ${ }^{2}$ Even more recently, identity-based encryption (IBE) schemes with "somewhat tight" security (under simple assumptions) have been constructed $[6,11]$. (This required new techniques, since it is not clear how to extend the techniques of $[1,18,20]$ to the IBE setting.) In this case, "somewhat tight" means that their security reduction loses only a small multiplicative factor, but still considers the standard IBE security experiment [9] with one encryption and one instance of the scheme. Nonetheless, while the IBE schemes from [6,11] are not proved tightly secure in a multi-user, multiciphertext setting, these schemes imply tightly secure PKE schemes (even in the multi-user, multi-ciphertext setting) when plugged into the transformations of $[9,18,20] .^{3}$

Our Contribution. In this work, we construct the first IBE scheme with an almost tight security reduction in the multi-instance, multi-ciphertext scenario. Our reduction is only almost tight, since it loses a factor of $\mathbf{O}(k)$, where $k$ is the security parameter. However, we stress that this loss is independent of the number of ciphertexts, revealed user secret keys, or instances of the scheme.

${ }^{1}$ A " $q$-type" assumption may depend on the size of the investigated cryptographic system. (That is, larger cryptographic systems may only be secure under a stronger instance of the assumption.) Hence, a tight reduction (even in a multi-instance scenario) to a $q$-type assumption may not yield security guarantees that are independent of the number of users.

${ }^{2}$ A "simple" assumption is defined through a security game in which an adversary first gets a challenge whose size only depends on the security parameter, and must then output a unique solution without further interaction. Examples of simple assumptions are DLOG, DDH, or RSA, but not Strong Diffie-Hellman [8] or $q$-ABDHE [15].

${ }^{3}$ More specifically, Boneh and Franklin [9] mention (and attribute this observation to Naor) that every IBE scheme can be viewed as a signature scheme. The signature schemes thus derived from $[6,11]$ are then suitable for the conversions of $[18,20]$, yielding PKE schemes tightly secure in the multi-user, multi-ciphertext setting. 
In our security reduction, we rely on a computational assumption in compositeorder pairing-friendly groups; this assumption is a variant of an assumption used by Chen and Wee [11] for their IBE scheme, and in particular simple in the above sense. We note that a conversion to the prime-order setting using the techniques from $[13,17,19,21]$ (see also [5]) seems plausible - specifically since Chen and Wee [11] already describe such a conversion for their assumption-, but we leave such a conversion as an open problem.

Our Approach. Our scheme is a variant of the IBE scheme by Chen and Wee [11] (which is almost tightly secure in the one-instance, one-ciphertext setting), and our proof strategy draws heavily from theirs. Hence, to describe our techniques, let us first briefly sketch their strategy.

In a nutshell, Chen and Wee start with a real security game, in which an adversary $A$ receives a master public key $m p k$ of the scheme, as well as access to arbitrarily many user secret keys $u s k_{i d}$ for adversarially chosen identities $i d$. At some point, $A$ selects a fresh challenge identity $i d^{*}$ and two messages $M_{0}^{*}, M_{1}^{*}$, and then receives the encryption $C_{i d^{*}}^{*} \leftarrow \operatorname{Enc}\left(m p k, i d^{*}, M_{b}\right)$ (under identity $i d^{*}$ ) of one of these messages. After potentially querying more user secret keys (for identities $\left.i d \neq i d^{*}\right), A$ eventually outputs a guess $b^{*}$ for $b$. If $b^{*}=b$, we say that $A$ wins. Chen and Wee then show security by gradually changing this game (being careful not to significantly decrease $A$ 's success), until $A$ trivially cannot win (except by guessing).

As a first preparatory change, Chen and Wee use the user secret key $u s k_{i d^{*}}$ to construct the challenge ciphertext $C_{i d^{*}}^{*}$. (This way, the encryption random coins for $C_{i d^{*}}^{*}$ do not have to be known to the security game.) Additionally $C_{i d^{*}}^{*}$ is now of a special, "pseudo-normal" form that will later enable a gradual randomization of the encrypted message. The core of the proof then consists of a number of hybrid steps, in which the distribution of all generated user secret keys (including the user secret key $u s k_{i d^{*}}$ used to generate $C_{i d^{*}}^{*}$ ) is modified. Concretely, in the $i$-th hybrid game, each used $u s k_{i d}$ contains an additional "blinding term" of the form $R\left(\left.i d\right|_{i}\right)$, where $\left.i d\right|_{i}$ is the $i$-bit prefix of $i d$, and $R$ is a truly random function. Eventually, each user secret key $u s k_{i d}$ will be fully randomized by a truly random value $R(i d)$. In particular, at this point, the key $u s k_{i d^{*}}$ used to prepare $C_{i d^{*}}^{*}$ is blinded by a fresh random value $R\left(i d^{*}\right)$. By the special "pseudo-normal" form of $C_{i d^{*}}^{*}$, this means that the corresponding encrypted message is also blinded, and $A$ 's view is finally independent of the challenge bit $b$.

We keep this high-level proof structure, extending it of course to multiple ciphertexts and multiple instances of the scheme. However, as we will explain below, the way Chen and Wee gradually introduce the blinding terms $R\left(\left.i d\right|_{i}\right)$ does not immediately extend to many ciphertexts or instances; hence, we need to deviate from their proof strategy here.

The Problem. Specifically, Chen and Wee move from the $(i-1)$-th to the $i$-th hybrid through a single reduction as follows: first, they guess the $i$-th bit $i d_{i}^{*}$ of the challenge identity $i d^{*}$. Then, they set up things such that 
(a) all user secret keys for identities $i d$ with $i d_{i}=i d_{i}^{*}$ (i.e., that coincide in the $i$-th bit with $i d^{*}$ ) behave as in the previous hybrid (i.e., carry a blinding term $\left.R\left(\left.i d\right|_{i-1}\right)\right)$,

(b) all user secret keys for identities $i d$ with $i d_{i}=1-i d_{i}^{*}$ carry a blinding term of $\left.R\left(\left.i d\right|_{i-1}\right) \cdot R^{\prime}\left(\left.i d\right|_{i-1}\right)\right)$. Depending on the input of the reduction, we have either that $R^{\prime}=1$ (such that the overall blinding term is $R\left(\left.i d\right|_{i-1}\right)$ ), or that $R^{\prime}$ is an independently random function. (In particular, all $u s k_{i d}$ with $i d_{i}=1-i d_{i}^{*}$ contain an embedded computational challenge $R^{\prime}$.)

Depending on whether or not $R^{\prime}=1$, this setup simulates the $(i-1)$-th or the $i$-th hybrid. However, we remark that the setup of Chen and Wee only allows to generate "pseudo-normal" challenge ciphertexts $C_{i d^{*}}^{*}$ for identities $i d^{*}$ with the initially guessed $i$-th bit $i d_{i}^{*}$. (Intuitively, any pseudo-normal ciphertext for an identity $i d$ with $i d_{i}=1-i d_{i}^{*}$ would "react with" an additional blinding term $R^{\prime}\left(\left.i d\right|_{i-1}\right)$ in $u s k_{i d}$, allowing to trivially solve the computational challenge.)

Hence, in their $i$-th game hop, only challenge ciphertexts for identities with the same $i$-th bit can be generated. Thus, their approach cannot in any obvious way be extended to multiple challenge ciphertexts for different identities. (For similar reasons, a generalization to multiple instances of the scheme fails.)

Our Solution. In order to move from the $(i-1)$-th to the $i$-th hybrid, we thus follow a different strategy that involves three reductions. The main technical ingredient in our case is the ability to distribute the blinding terms $R\left(\left.i d\right|_{i}\right)$ in user secret keys into two different "compartments" (i.e., subgroups) of the composite-order group we are working in. (In particular, a term $R\left(\left.i d\right|_{i}\right)$ in one compartment can be changed independently of terms in the other compartment.)

More specifically, recall that in the $(i-1)$-th hybrid, all user secret keys carry an additional $R\left(\left.i d\right|_{i-1}\right)$ blinding term, and all challenge ciphertexts are pseudo-normal (in the sense that they "react with" the blinding terms in user secret keys). In our first step, we move all blinding terms $R\left(\left.i d\right|_{i-1}\right)$ in the $u s k_{i d}$ into the two compartments, depending on the $i$-th bit of $i d$. (That is, if $i d_{i}=0$, then the corresponding blinding term $R\left(\left.i d\right|_{i-1}\right)$ goes into the first compartment, and if $i d_{i}=1$, then it goes into the second.)

In our second step, we can now treat the embedded blinding terms for $i d_{i}=0$ and $i d_{i}=1$ separately. In particular, since these cases are now "decoupled" by being in different compartments, we can completely re-randomize the underlying random function $R$ in exactly one of those compartments. (This does not lead to trivial distinctions of the computational challenge since we do not introduce new blinding terms that would "react with" pseudo-normal ciphertexts and thus become easily detectable. Instead, we simply decouple existing blinding terms in different subgroups.) Note however that since now different random functions, say, $\widehat{R}$ and $\widetilde{R}$, determine the blinding terms used for identities with $i d_{i}=0$ and $i d_{i}=1$, we essentially obtain blinding terms that depend on the first $i$ (and not only $i-1$ ) bits of $i d$.

Finally, we revert the first change and move all blinding terms in the $u s k_{i d}$ into one compartment. In summary, this series of three moves has thus created blinding terms that depend on the first $i$ bits of $i d$. Thus, we have moved to 
the $i$-th hybrid. If we follow the high-level strategy of Chen and Wee again, this yields a sequence of $\mathbf{O}(k)$ reductions that show the security of our IBE scheme. (From a conceptual perspective, it might also be interesting to note that none of our reductions needs to guess, e.g., an identity bit.)

Outline of the Paper. After introducing some preliminary definitions in Section 2, we explain the necessary algebraic structure (mentioned in the "compartment discussion" above) of "extended nested dual system groups" (ENDSGs) in Section 3. (This structure extends a similar structure of Chen and Wee [11].) In Section 4, we present our IBE scheme from ENDSGs, and in Section 5, we show how to instantiate ENDSGs in composite-order pairing-friendly groups.

\section{Preliminaries}

Notation. For $n \in \mathbb{N}$, let $[n]:=\{1, \ldots, n\}$, and let $k \in \mathbb{N}$ be the security parameter. For a finite set $\mathcal{S}$, we denote by $s \leftarrow \mathcal{S}$ the process of sampling $s$ uniformly from $\mathcal{S}$. For an algorithm $A$, let $y \leftarrow A(k, x)$ be the process of running $A$ on input $k, x$ with access to uniformly random coins and assigning the result to $y$. (We may omit to mention the $k$-input explicitly and assume that all algorithms take $k$ as input.) To make the random coins $r$ explicit, we write $A(k, x ; r)$. We say an algorithm $A$ is probabilistic polynomial time (PPT) if the running time of $A$ is polynomial in $k$. A function $f: \mathbb{N} \rightarrow \mathbb{R}$ is negligible if it vanishes faster than the inverse of any polynomial (i.e., if $\forall c \exists k_{0} \forall k \geq k_{0}:|f(k)| \leq 1 / k^{c}$ ). Further, we write vectors in bold font, e.g., $\mathbf{v}=\left(v_{1}, \ldots, v_{n}\right)$ for a vectors of length $n \in \mathbb{N}$ and with components $v_{1}, \ldots, v_{n}$. (We may also write $\mathbf{v}=\left(v_{i}\right)_{i \in[n]}$ or even $\mathbf{v}=\left(v_{i}\right)_{i}$ in this case.) In the following, we use a component-wise multiplication of vectors, i.e., $\mathbf{v} \cdot \mathbf{v}^{\prime}=\left(v_{1}, \ldots, v_{n}\right) \cdot\left(v_{1}^{\prime}, \ldots, v_{n}^{\prime}\right)=\left(v_{1} \cdot v_{1}^{\prime}, \ldots, v_{n} \cdot v_{n}^{\prime}\right)$. Further, we write $\mathbf{v}^{j}:=\left(v_{1}^{j}, \ldots, v_{n}^{j}\right)$, for $j \in \mathbb{N}$, and $\mathbf{v}_{-i}:=\left(v_{1}, \ldots, v_{i-1}, v_{i+1}, \ldots, v_{n}\right)$, for $i \in[n]$, and $s^{\mathbf{v}}:=\left(s^{v_{1}}, \ldots, s^{v_{n}}\right)$. For two random variables $X, Y$, we denote with $\mathrm{SD}(X ; Y)$ is the statistical distance of $X$ and $Y$.

Identity-Based Encryption. An identity-based encryption (IBE) scheme IBE with identity space $\mathcal{I D}$ and message space $\mathcal{M}$ consists of the five PPT algorithms Par, Gen, Ext, Enc, Dec. Parameter sampling Par $(k)$, on input a security parameter $k$, outputs public parameters $p p$ and secret parameters $s p$. Key generation $\operatorname{Gen}(p p, s p)$, on input $p p$ and $s p$, outputs a master public key $m p k$ and a master secret key $m s k$. User secret key extraction Ext $(m s k, i d)$, given $m s k$ and an identity $i d \in \mathcal{I D}$, outputs a user secret key $u s k_{i d}$ associated with $i d$. Encryption $\operatorname{Enc}(m p k, i d, M)$, given $m p k$, an identity $i d \in \mathcal{I} \mathcal{D}$, and a message $M \in \mathcal{M}$, outputs an $i d$-associated ciphertext $C_{i d}$. Decryption $\operatorname{Dec}\left(u s k_{i d}, C_{i d}\right)$, given $u s k_{i d}$ for an identity $i d$, and ciphertext $C_{i d}$, outputs $M \in \mathcal{M} \cup\{\perp\}$. For correctness, we require that for any $k \in \mathbb{N}$, for all $(p p, s p) \leftarrow \operatorname{Par}(k)$, for all $(m p k, m s k) \leftarrow \operatorname{Gen}(p p, s p)$, for all $i d \in \mathcal{I D}$, for all $u s k_{i d} \leftarrow \operatorname{Ext}(m s k, i d)$, for all $M \in \mathcal{M}$, and for all $C_{i d} \leftarrow \operatorname{Enc}(m p k, i d, M)$, Dec satisfies $\operatorname{Dec}\left(u s k_{i d}, C_{i d}\right)=M$. For security, we define multi-instance, multi-ciphertext IBE security, dubbed $(\mu, q)$-IBE-IND-CPA security, for $(\mu, q) \in \mathbb{N}^{2}$, as follows. 


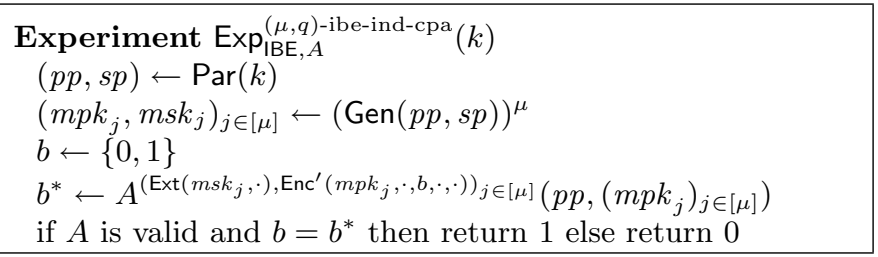

Fig. 1. The $(\mu, q)$-IBE-IND-CPA security experiment

(Weak) $(\mu, q)$-IBE-IND-CPA Security. An IBE scheme IBE defined as above is $(\mu, q)$-IBE-IND-CPA-secure if and only if any PPT adversary $A$ succeeds in the following experiment only with probability at most negligibly larger than $1 / 2$. Let $\operatorname{Enc}^{\prime}\left(m p k, i d, b, M_{0}, M_{1}\right)$ be a PPT auxiliary encryption oracle that, given a master public key $m p k$, a challenge identity $i d \in \mathcal{I D}$, a bit $b \in\{0,1\}$, and two messages $M_{0}, M_{1} \in \mathcal{M}$, outputs a challenge ciphertext $C_{i d} \leftarrow \operatorname{Enc}\left(m p k, i d, M_{b}\right)$. First, $A$ gets honestly generated public parameter $p p$ and master public keys $\left(m p k_{1}, \ldots, m p k_{\mu}\right)$. During the experiment, $A$ may adaptively query $\operatorname{Ext}\left(m s k_{j}, \cdot\right)$ oracles and $\operatorname{Enc}^{\prime}\left(m p k_{j}, \cdot, b, \cdot, \cdot\right)$-oracles, for corresponding $m p k_{j}, m s k_{j}$ and a (uniform) bit $b \leftarrow\{0,1\}$, for all $j \in[\mu]$. Eventually, $A$ outputs a guess $b^{*}$. We say that $A$ is valid if and only if $A$ never queries an $\operatorname{Ext}\left(m s k_{j}, \cdot\right)$ oracle on an identity $i d$ for which it has already queried the corresponding $\operatorname{Enc}^{\prime}\left(m p k_{j}, \cdot, b, \cdot, \cdot\right)$ oracle (and vice versa); each message pair $A$ selected as input to Enc' contained only equal-length messages; and $A$ has only queried its Enc'-oracles at most $q$ times per $j$-instance. We say that $A$ succeeds if and only if $A$ is valid and $b=b^{*}$. Concretely, the previous described experiment is given in Figure 1 and denoted $\operatorname{Exp}_{\mathrm{IBE}, A}^{(\mu, q) \text {-ibe-ind-cpa }}$. Further, we define the advantage function for any $\mathrm{PPT} A$ as $\operatorname{Adv}_{\mathrm{IBE}, A}^{(\mu, q) \text {-ibe-ind-cpa }}(k):=\left|\operatorname{Pr}\left[\operatorname{Exp}_{\mathrm{IBE}, A}^{(\mu, q) \text {-ibe-ind-cpa }}(k)=1\right]-1 / 2\right|$.

Furthermore, we call IBE weakly $(\mu, q)$-IBE-IND-CPA secure if and only if $\operatorname{Adv}_{\mathrm{IBE}, A}^{(\mu, q) \text {-ibe-ind-cpa }}$ is negligible for all weak $\mathrm{PPT}$ adversaries $A$. Here, $A$ is weak if it never requests challenge ciphertexts for the same scheme instance and identity twice (i.e., if it never queries any $\operatorname{Enc}^{\prime}\left(m p k_{j}, \cdot, b, \cdot, \cdot\right)$ oracle twice with the same identity $i d)$.

Finally, we remark that the one-instance, one-ciphertext notion $(1,1)$-IBEIND-CPA is the standard notion of IBE security considered in, e.g., $[6,9,11]$.

Pairings. Let $G, H, G_{T}$ be cyclic groups of order $N$. A pairing e $: G \times H \rightarrow$ $G_{T}$ is a map that is bilinear (i.e., for all $g, g^{\prime} \in G$ and $h, h^{\prime} \in H$, we have $e\left(g \cdot g^{\prime}, h\right)=e(g, h) \cdot e\left(g^{\prime}, h\right)$ and $\left.e\left(g, h \cdot h^{\prime}\right)=e(g, h) \cdot e\left(g, h^{\prime}\right)\right)$, non-degenerate (i.e., for generators $g \in G, h \in H$, we have that $e(g, h) \in G_{T}$ is a generator), and efficiently computable.

\section{$3 \quad$ Extended Nested Dual System Groups}

(Nested) Dual System Groups. Nested dual system groups (NDSG) [11] can be seen as a variant of dual system groups (DSG) [12] which itself are based 
on the dual system framework introduced by Waters [21]. NDSGs were recently defined by Chen and Wee and enabled to prove the first IBE (almost) tightly and fully secure under simple assumptions. In the following, based on NDSGs, we construct a new notion we call extended nested dual system groups.

A Variant of Nested Dual System Groups. We introduce a variant of Chen and Wee's nested dual system groups (NDSG) [11], dubbed extended NDSG (ENDSG). (Mainly, we re-use and extend the notions from [11].) Further, let $\mathrm{G}\left(k, n^{\prime}\right)$ be a group generator that, given integers $k$ and $n^{\prime}$, generates the tuple $\left(G, H, G_{T}, N,\left(g_{p_{1}}, \ldots, g_{p_{n^{\prime}}}\right),\left(h_{p_{1}}, \ldots, h_{p_{n^{\prime}}}\right), g, h, e\right)$, for a pairing $e: G \times H \rightarrow G_{T}$, for composite-order groups $G, H, G_{T}$, all of known group order $N=p_{1} \cdots p_{n^{\prime}}$, for $k$-bit primes $\left(p_{i}\right)_{i}$. Further, $g$ and $h$ are generators of $G$ and $H$, and $\left(g_{p_{i}}\right)_{i}$ and $\left(h_{p_{i}}\right)_{i}$ are generators of the (proper) subgroups $G_{p_{i}} \subset G$ and $H_{p_{i}} \subset H$ of order $\left|G_{p_{i}}\right|=\left|H_{p_{i}}\right|=p_{i}$, respectively. In this setting, an ENDSG ENDSG consists of algorithms SampP, SampG, SampH, $\widehat{\operatorname{SampG}}, \widehat{\operatorname{SampG}}$

Parameter sampling. SampP $(k, n)$, given security parameter $k$ and parameter $n \in \mathbb{N}$, samples $\left(G, H, G_{T}, N,\left(g_{p_{1}}, \ldots, g_{p_{n^{\prime}}}\right),\left(h_{p_{1}}, \ldots, h_{p_{n^{\prime}}}\right), g, h, e\right) \leftarrow$ $\mathrm{G}\left(k, n^{\prime}\right)$, for an integer $n^{\prime}$ determined by SampP, and outputs public parameters $p p=\left(G, H, G_{T}, N, g, h, e, m, n\right.$, pars $)$ and secret parameters $s p=(\widehat{h}, \widetilde{h}$, $\widehat{p a r s}, \widehat{p a r s})$, where $m: H \rightarrow G_{T}$ is a linear map, $\widehat{h}, \widetilde{h}$ are nontrivial $H$ elements, and pars, $\widehat{p a r s}, \widetilde{p a r s}$ may contain arbitrary additional information used by SampG, SampH, and $\widehat{\operatorname{SampG}}$ and $\widehat{\text { SampG }}$.

$G$-group sampling. SampG $(p p)$, given $p p$, outputs $\mathbf{g}=\left(g_{0}, \ldots, g_{n}\right) \in G^{n+1}$. $H$-group sampling. $\operatorname{SampH}(p p)$, given $p p$, outputs $\mathbf{h}=\left(h_{0}, \ldots, h_{n}\right) \in H^{n+1}$.

Semi-functional $G$-group sampling 1. $\widehat{\operatorname{SampG}}(p p, s p)$, given $p p$ and $s p$, outputs $\widehat{\mathrm{g}}=\left(\widehat{g}_{0}, \ldots, \widehat{g}_{n}\right) \in G^{n+1}$.

Semi-functional $G$-group sampling 2. $\overparen{\operatorname{SampG}}(p p, s p)$, given $p p$ and $s p$, outputs $\widetilde{\mathbf{g}}=\left(\widetilde{g}_{0}, \ldots, \widetilde{g}_{n}\right) \in G^{n+1}$.

Correctness of ENDSG. For correctness, for all $k \in \mathbb{N}$, for all integers $n=$ $n(k)>1$, for all $p p$, where $p p$ is the first ouput of $\operatorname{SampP}(k, n)$, we require:

Associativity. For all $\left(g_{0}, \ldots, g_{n}\right) \leftarrow \operatorname{SampG}(p p)$ and for all $\left(h_{0}, \ldots, h_{n}\right) \leftarrow$ SampH $(p p)$, we have $e\left(g_{0}, h_{i}\right)=e\left(g_{i}, h_{0}\right)$, for all $i$.

Projective. For all $s \leftarrow \mathbb{Z}_{N}^{*}$, for all $g_{0}$ which is the first output of SampG $(p p ; s)$, for all $h \in H$, we have $m(h)^{s}=e\left(g_{0}, h\right)$.

Security of ENDSG. For security, for all $k \in \mathbb{N}$, for all integers $n=n(k)>1$, for all $(p p, s p) \leftarrow \operatorname{SampP}(k, n)$, we require:

Orthogonality. For $m$ specified in $p p$, for $\widehat{h}, \widetilde{h}$ specified in $s p$, we have $m(\widehat{h})=$ $m(\widetilde{h})=1$. For $g_{0}, \widehat{g}_{0}$, and $\widetilde{g}_{0}$ that are the first outputs of SampG $(p p)$, $\widehat{\mathrm{SampG}}(p p, s p)$, and $\widehat{\mathrm{SampG}}(p p, s p)$, respectively, we have that $e\left(g_{0}, \widehat{h}\right)=1$, $e\left(g_{0}, \widetilde{h}\right)=1, e\left(\widehat{g}_{0}, \widetilde{h}\right)=1$, and $e\left(\widetilde{g}_{0}, \widehat{h}\right)=1$. 
$G$ - and $H$-subgroups. The outputs of SampG, $\widehat{\text { SampG }}$, and $\widetilde{\text { SampG }}$ are distributed uniformly over the generators of different nontrivial subgroups of $G^{n+1}$ (that only depend on $p p$ ) of coprime order, respectively, while the output of SampH is uniformly distributed over the generators of a nontrivial subgroup of $H^{n+1}$ (that only depends on $p p$ ).

Non-degeneracy. For $\widehat{h}$ specified in $s p$ and for $\widehat{g}_{0}$ which is the first output of $\widehat{\mathrm{SampG}}(p p, s p)$, it holds that $e\left(\widehat{g}_{0}, \widehat{h}\right)$ is uniformly distributed over the generators of a nontrivial subgroup of $G_{T}$ (that only depends on $p p$ ). Similarly, $e\left(\widetilde{g}_{0}, \widetilde{h}\right)$ is uniformly distributed over the generators of a nontrivial subgroup of $G_{T}$ (that only depends on $p p$ ), where $\widetilde{h}$ is specified in $s p$ and $\widetilde{g}_{0}$ is the first output of SampG $(p p, s p)$.

Left-subgroup indistinguishability 1 (LS1). For any PPT adversary $D$, we have that the function

$$
\operatorname{Adv}_{\mathrm{ENDSG}, \mathrm{G}, D}^{\mathrm{ls} 1}(k, n):=|\operatorname{Pr}[D(p p, \mathbf{g})=1]-\operatorname{Pr}[D(p p, \mathbf{g} \widehat{\mathbf{g}})=1]|
$$

is negligible in $k$, where $\mathbf{g} \leftarrow \operatorname{SampG}(p p), \widehat{\mathbf{g}} \leftarrow \widehat{\operatorname{SampG}}(p p, s p)$.

Left-subgroup indistinguishability 2 (LS2). For any PPT adversary $D$, we have that the function

$$
\begin{aligned}
\operatorname{Adv}_{\mathrm{ENDSG}, \mathrm{G}, D}^{\mathrm{ls} 2}(k, n):= & \mid \operatorname{Pr}\left[D\left(p p, \widehat{h} \widetilde{h}, \mathbf{g}^{\prime} \widehat{\mathbf{g}}^{\prime}, \mathbf{g} \widehat{\mathbf{g}}\right)=1\right] \\
& -\operatorname{Pr}\left[D\left(p p, \widehat{h} \widetilde{h}, \mathbf{g}^{\prime} \widehat{\mathbf{g}}^{\prime}, \mathbf{g} \widetilde{\mathbf{g}}\right)=1\right] \mid
\end{aligned}
$$

is negligible in $k$, where $\mathbf{g}, \mathbf{g}^{\prime} \leftarrow \operatorname{SampG}(p p), \widehat{\mathbf{g}}, \widehat{\mathbf{g}}^{\prime} \leftarrow \widehat{\operatorname{SampG}}(p p, s p), \widetilde{\mathbf{g}} \leftarrow$ $\widehat{\operatorname{SampG}}(p p, s p)$, for $\widehat{h}$ and $\widetilde{h}$ specified in $s p$.

Nested-hiding indistinguishability (NH). For any PPT adversary $D$, for all integers $q^{\prime}=q^{\prime}(k)$, the function

$$
\begin{aligned}
& \operatorname{Adv}_{\mathrm{ENDSG}, \mathrm{G}, D}^{\mathrm{nh}}\left(k, n, q^{\prime}\right):= \\
& \max _{i \in\left[\left\lfloor\frac{n}{2}\right\rfloor\right]}\left(\mid \operatorname{Pr}\left[D\left(p p, \widehat{h}, \widetilde{h}, \widehat{\mathbf{g}}_{-(2 i-1)}, \widetilde{\mathbf{g}}_{-2 i},\left(\mathbf{h}_{1}, \ldots, \mathbf{h}_{q^{\prime}}\right)\right)=1\right]\right. \\
& \left.-\operatorname{Pr}\left[D\left(p p, \widehat{h}, \widetilde{h}, \widehat{\mathbf{g}}_{-(2 i-1)}, \widetilde{\mathbf{g}}_{-2 i},\left(\mathbf{h}_{1}^{\prime}, \ldots, \mathbf{h}_{q^{\prime}}^{\prime}\right)\right)=1\right] \mid\right),
\end{aligned}
$$

is negligible in $k$, where $\widehat{\mathbf{g}} \leftarrow \widehat{\operatorname{SampG}}(p p, s p), \widetilde{\mathbf{g}} \leftarrow \widehat{\operatorname{SampG}}(p p, s p)$, and

$$
\begin{gathered}
\mathbf{h}_{i^{\prime}}:=\left(h_{i^{\prime}, 0}, \ldots, h_{i^{\prime}, n}\right) \leftarrow \operatorname{SampH}(p p), \\
\mathbf{h}_{i^{\prime}}^{\prime}:=\left(h_{i^{\prime}, 0}, \ldots, h_{i^{\prime}, 2 i-1} \cdot(\widehat{h})^{\widehat{\gamma}_{i^{\prime}}}, h_{i^{\prime}, 2 i} \cdot(\widetilde{h})^{\widetilde{\gamma}_{i^{\prime}}}, \ldots, h_{i^{\prime}, n}\right),
\end{gathered}
$$

for $\widehat{h}, \widetilde{h}$ specified in $s p$, for $\widehat{\gamma}_{i^{\prime}}, \widetilde{\gamma}_{i^{\prime}} \leftarrow \mathbb{Z}_{\text {ord }(H)}^{*}$, and for all $i^{\prime} \in\left[q^{\prime}\right]$.

(Informal) Comparison of NDSGs and ENDSGs. Loosely speaking, in contrast to the NDSGs from [11], ENDSGs have a second semi-functional $G$ group sampling algorithm SampG as well as a second nontrivial $H$-element in $s p$ (i.e., $\widetilde{h})$. Further, we omit the SampGT-algorithm. Concerning the ENDSG properties, we extend the NDSG properties and assumptions appropriately and introduce one additional assumption (i.e., LS2). 


\section{An (Almost) Tightly $(\mu, q)$-IBE-IND-CPA-Secure IBE}

A Variant of the IBE of Chen and Wee [11]. We are now ready to present our variant of Chen and Wee's IBE scheme [11]. We use an ENDSG ENDSG $=($ SampP, SampG, SampH, $\widehat{\text { SampG }}, \widehat{\text { SampG }})$ from Section 3 as a basic building block. Besides, for groups $G_{T}$ (defined below), let $\mathcal{U H}$ be a family of universal hash functions $\mathrm{H}: G_{T} \rightarrow\{0,1\}^{k}$ such that for any nontrivial subgroup $G_{T}^{\prime} \subset G_{T}$, and for $\mathrm{H} \leftarrow \mathcal{U H}, X \leftarrow G_{T}^{\prime}$, and $U \leftarrow\{0,1\}^{k}$, we have $\mathrm{SD}((\mathrm{H}, \mathrm{H}(X)) ;(\mathrm{H}, U))=\mathbf{O}\left(2^{-k}\right)$. Let IBE $=($ Par, Gen, Ext, Enc, Dec $)$ with identity space $\mathcal{I D}=\{0,1\}^{n}$ and message space $\mathcal{M}=\{0,1\}^{k}$ be defined as follows:

Parameter Generation. $\operatorname{Par}(k, n)$ samples $\left(p p^{\prime}, s p^{\prime}\right) \leftarrow \operatorname{SampP}(k, 2 n)$, with $p p^{\prime}=\left(G, H, G_{T}, N, g, h, e, m, 2 n\right.$, pars $)$ and $\left.s p^{\prime}=(\widehat{h}, \widetilde{h}, \widehat{p a r s}, \widehat{p a r s})\right)$, and $\mathrm{H} \leftarrow \mathcal{U H}$, and then outputs the public and secret parameters $(p p, s p)$, where $p p=\left(G, H, G_{T}, N, g, h, e, m, 2 n, \mathrm{H}\right.$, pars $)$ and $s p=s p^{\prime}$.

Key Generation. Gen $(p p, s p)$ samples $m s k \leftarrow H$, and outputs a master public key $m p k:=(p p, m(m s k))$ and a master secret key $m s k$.

Secret-Key Extraction. Ext $(m s k, i d)$, given $m s k \in H$ and an identity $i d=$ $\left(i d_{1} \ldots i d_{n}\right) \in \mathcal{I D}$, samples $\left(h_{0}, \ldots, h_{2 n}\right) \leftarrow \operatorname{SampH}(p p)$ and outputs a user secret key

$$
u s k_{i d}:=\left(h_{0}, m s k \cdot \prod_{i=1}^{n} h_{2 i-i d_{i}}\right) .
$$

Encryption. Enc $(m p k, i d, M)$, given $m p k=(p p, m(m s k))$, an identity $i d=$ $\left(i d_{1} \ldots i d_{n}\right) \in \mathcal{I D}$, and a message $M \in \mathcal{M}$, computes $\left(g_{0}, \ldots, g_{2 n}\right):=$ $\operatorname{SampG}(p p ; s)$, for $s \leftarrow \mathbb{Z}_{N}^{*}$, and $g_{T}:=m(m s k)^{s}\left(=e\left(g_{0}, m s k\right)\right)$, and outputs a ciphertext

$$
C_{i d}:=\left(g_{0}, \prod_{i=1}^{n} g_{2 i-i d_{i}}, \mathrm{H}\left(g_{T}\right) \oplus M\right) .
$$

Decryption. $\operatorname{Dec}\left(u s k_{i d}, C_{i d^{\prime}}\right)$, given a user secret key $u s k_{i d}=:\left(K_{0}, K_{1}\right)$ and a ciphertext $C_{i d^{\prime}}=:\left(C_{0}, C_{1}, C_{2}\right)$, outputs

$$
M:=\mathrm{H}\left(\frac{e\left(C_{0}, K_{1}\right)}{e\left(C_{1}, K_{0}\right)}\right) \oplus C_{2} .
$$

Correctness of IBE. We have

$$
\begin{aligned}
\mathrm{H}\left(\frac{e\left(C_{0}, K_{1}\right)}{e\left(C_{1}, K_{0}\right)}\right) \oplus C_{2} & =\mathrm{H}\left(\frac{e\left(g_{0}, m s k \cdot \prod_{i=1}^{n} h_{2 i-i d_{i}}\right)}{e\left(\prod_{i=1}^{n} g_{2 i-i d_{i}^{\prime}}, h_{0}\right)}\right) \oplus \mathrm{H}\left(g_{T}\right) \oplus M \\
& \stackrel{(*)}{=} \mathrm{H}\left(g_{T}\right) \oplus \mathrm{H}\left(g_{T}\right) \oplus M,
\end{aligned}
$$

for $i d=i d^{\prime} .(*)$ holds due to ENDSG's associativity and projective properties. $(\mu, q)$-IBE-IND-CPA Security of IBE. We base our high-level proof strategy on the IBE-IND-CPA proof strategy of Chen and Wee [11], but deviate on 
the low level. First, we define auxiliary encryption $\overline{\text { Enc }}$ and auxiliary secret-key extraction $\overline{\mathrm{Ext}}$, random functions $\widehat{\mathrm{R}}_{j, i}$ and $\widetilde{\mathrm{R}}_{j, i}$, pseudo-normal ciphertexts, semifunctional type- $(\cdot, i)$ ciphertexts, and semi-functional type- $i$ user secret keys as in [11]:

Auxiliary Secret-Key Extraction. $\overline{\operatorname{Ext}}(p p, m s k, i d ; \mathbf{h})$, given $p p$, master secret key $m s k$, an identity $i d=i d_{1} \ldots i d_{n} \in \mathcal{I D}$, and $\mathbf{h}=\left(h_{0}, \ldots, h_{2 n}\right) \in$ $(H)^{2 n+1}$, outputs a user secret key

$$
u s k_{i d}:=\left(h_{0}, m s k \cdot \prod_{i=1}^{n} h_{2 i-i d_{i}}\right) .
$$

Auxiliary Encryption Function. $\overline{\mathrm{Enc}}(p p, i d, M ; m s k, \mathbf{g})$, given parameter $p p$, identity $i d=i d_{1} \ldots i d_{n} \in \mathcal{I D}$, message $M \in \mathcal{M}$, master secret key $m s k$, and $\mathbf{g}=\left(g_{0}, \ldots, g_{2 n}\right) \in(G)^{2 n+1}$, outputs a ciphertext

$$
C_{i d}:=\left(g_{0}, \prod_{i=1}^{n} g_{2 i-i d_{i}}, \mathrm{H}\left(e\left(g_{0}, m s k\right)\right) \oplus M\right) .
$$

Random Function Families. Let $\left.i d\right|_{i}:=i d_{1} \ldots i d_{i}$ be the $i$-bit prefix of an identity $i d$, and let $\left.\mathcal{I D}\right|_{i}:=\{0,1\}^{i}$. For an instance $j$ and $i \in[n] \cup\{0\}$, consider functions $\widehat{\mathrm{R}}_{j, i}:\left.\mathcal{I D}\right|_{i} \rightarrow H,\left.i d\right|_{i} \mapsto(\widehat{h})^{\widehat{\gamma}_{j, i}\left(\left.i d\right|_{i}\right)}$ and $\widetilde{\mathrm{R}}_{j, i}:\left.\mathcal{I D}\right|_{i} \rightarrow$ $H,\left.\quad i d\right|_{i} \mapsto(\widetilde{h})^{\widetilde{\gamma}_{j, i}\left(\left.i d\right|_{i}\right)}$, where $\widehat{\gamma}_{j, i}:\left.\mathcal{I D}\right|_{i} \rightarrow \mathbb{Z}_{\text {ord }(H)},\left.i d\right|_{i} \mapsto \widehat{\gamma}_{j,\left.i d\right|_{i}}$ and $\widetilde{\gamma}_{j, i}$ : $\left.\mathcal{I D}\right|_{i} \rightarrow \mathbb{Z}_{\text {ord }(H)}^{*},\left.i d\right|_{i} \mapsto \widetilde{\gamma}_{j,\left.i d\right|_{i}}$ are independently and truly random.

Pseudo-normal Ciphertexts. Pseudo-normal ciphertexts are generated as

$$
\begin{aligned}
C_{i d} & :=\overline{\mathrm{Enc}}(p p, i d, M ; m s k, \mathbf{g} \widehat{\mathrm{g}}) \\
& =\left(g_{0} \widehat{g}_{0}, \prod_{i=1}^{n} g_{2 i-i d_{i}} \widehat{g}_{2 i-i d_{i}}, \mathrm{H}\left(e\left(g_{0} \widehat{g}_{0}, m s k\right)\right) \oplus M\right),
\end{aligned}
$$

for uniform $\mathbf{g}=\left(g_{0}, \ldots, g_{2 n}\right) \leftarrow \operatorname{SampG}(p p)$ and $\widehat{\mathbf{g}}=\left(\widehat{g}_{0}, \ldots, \widehat{g}_{2 n}\right) \leftarrow$ $\widehat{\operatorname{SampG}}(p p, s p)$. (Hence, pseudo-normal ciphertexts have semi-functional $G$ components sampled from $\widehat{\operatorname{SampG}}$.)

Semi-functional type- $(\wedge, i)$ and type- $(\sim, i)$ Ciphertexts. Let $\widehat{\mathrm{R}}_{j, i}$ and $\widetilde{\mathrm{R}}_{j, i}$ be random functions as defined above. Semi-functional ciphertexts of type $(\wedge, i)$ are generated as

$$
\begin{aligned}
\widehat{C}_{i d} & :=\overline{\operatorname{Enc}}\left(p p, i d, M ; m s k \cdot \widehat{\mathrm{R}}_{j, i}\left(\left.i d\right|_{i}\right) \cdot \widetilde{\mathrm{R}}_{j, i}\left(\left.i d\right|_{i}\right), \mathbf{g} \widehat{\mathbf{g}}\right) \\
& \stackrel{(1)}{=}\left(g_{0} \widehat{g}_{0}, \prod_{i=1}^{n} g_{2 i-i d_{i}} \widehat{g}_{2 i-i d_{i}}, \mathrm{H}\left(e\left(g_{0} \widehat{g}_{0}, m s k \cdot \widehat{\mathrm{R}}_{j, i}\left(\left.i d\right|_{i}\right)\right)\right) \oplus M\right)
\end{aligned}
$$

while semi-functional ciphertexts of type $(\sim, i)$ are generated as

$$
\begin{aligned}
\widetilde{C}_{i d} & :=\overline{\mathrm{Enc}}\left(p p, i d, M ; m s k \cdot \widehat{\mathrm{R}}_{j, i}\left(\left.i d\right|_{i}\right) \cdot \widetilde{\mathrm{R}}_{j, i}\left(\left.i d\right|_{i}\right), \mathbf{g} \widetilde{\mathrm{g}}\right) \\
& \stackrel{(2)}{=}\left(g_{0} \widetilde{g}_{0}, \prod_{i=1}^{n} g_{2 i-i d_{i}} \widetilde{g}_{2 i-i d_{i}}, \mathrm{H}\left(e\left(g_{0} \widetilde{g}_{0}, m s k \cdot \widetilde{\mathrm{R}}_{j, i}\left(\left.i d\right|_{i}\right)\right)\right) \oplus M\right),
\end{aligned}
$$


where $\mathbf{g}=\left(g_{0}, \ldots, g_{2 n}\right) \leftarrow \operatorname{SampG}(p p), \widehat{\mathbf{g}}=\left(\widehat{g}_{0}, \ldots, \widehat{g}_{2 n}\right) \leftarrow \widehat{\operatorname{SampG}}(p p)$, and $\widetilde{\mathrm{g}}=\left(\widetilde{g}_{0}, \ldots, \widetilde{g}_{2 n}\right) \leftarrow \widehat{\operatorname{SampG}}(p p)$, while (1) and (2) hold due to ENDSG's properties.

Semi-functional type- $i$ User Secret Keys. Let $\widehat{\mathrm{R}}_{j, i}$ and $\widetilde{\mathrm{R}}_{j, i}$ be defined as above. For $\mathbf{h}=\left(h_{0}, \ldots, h_{2 n}\right) \leftarrow \operatorname{SampH}(p p)$, semi-functional type- $i$ user secret keys are generated as

$$
\begin{aligned}
u s k_{i d} & :=\overline{\operatorname{Ext}}\left(p p, m s k \cdot \widehat{\mathrm{R}}_{j, i}\left(\left.i d\right|_{i}\right) \cdot \widetilde{\mathrm{R}}_{j, i}\left(\left.i d\right|_{i}\right), i d ; \mathbf{h}\right) \\
& =\left(h_{0}, m s k \cdot \widehat{\mathrm{R}}_{j, i}\left(\left.i d\right|_{i}\right) \cdot \widetilde{\mathrm{R}}_{j, i}\left(\left.i d\right|_{i}\right) \cdot \prod_{i=1}^{n} h_{2 i-i d_{i}}\right) .
\end{aligned}
$$

Theorem 1. If ENDSG is a correct and secure ENDSG, then IBE defined as above is weakly $(\mu, q)$-IBE-IND-CPA-secure. Concretely, for any weak PPT adversary $A$ with at most $q^{\prime}=q^{\prime}(k)$ extraction queries per instance and running time $t$ in the $(\mu, q)-I B E-I N D-C P A$ security experiment with IBE, there are distinguishers $D_{1}$ on LS1, $D_{2}$ on LS2, and $D_{3}$ on $N H$ with running times $t_{1}^{\prime} \approx t_{2}^{\prime} \approx t_{3}^{\prime} \approx t+\mathbf{O}\left(\mu q q^{\prime} n k^{c}\right)$, respectively, some constant $c \in \mathbb{N}$, with

$$
\begin{aligned}
\operatorname{Adv}_{\mathrm{IBE}, A}^{(\mu, q) \text {-ibe-ind-cpa }}(k, n) \leq & \operatorname{Adv}_{\mathrm{ENDSG}, \mathrm{G}, D_{1}}^{\mathrm{ls} 1}(k, 2 n)+2 n \cdot \operatorname{Adv}_{\mathrm{ENDSG}, \mathrm{G}, D_{2}}^{\mathrm{Is} 2}(k, 2 n) \\
& +n \cdot \operatorname{Adv}_{\mathrm{ENDSG}, \mathrm{G}, D_{3}}^{\text {nh }}\left(k, 2 n, q^{\prime}\right)+\mu q \cdot \mathbf{O}\left(2^{-k}\right)
\end{aligned}
$$

for group generator $\mathrm{G}$ defined as above.

Proof. We show the $(\mu, q)$-IBE-IND-CPA security of IBE for any weak PPT adversary $A$ in a sequence of games where we successively change the games until we arrive at a game where $A$ has only negligible advantage (i.e., success probability of $1 / 2)$ in the sense of $(\mu, q)$-IBE-IND-CPA. Let $S_{A, j}$ be the event that $A$ succeeds in Game $j$. We give an overview how the challenge ciphertexts and user secret keys are generated in Table 1.

Game 0. Game 0 is the $(\mu, q)$-IBE-IND-CPA experiment as defined above.

Game 1. Game 1 is defined as Game 0 apart from the fact that all challenge ciphertexts are pseudo-normal.

Game 2.i.0. Game 2.i.0 is defined as Game 1 except that all user secret keys are semi-functional of type $(i-1)$ and all challenge ciphertexts are semifunctional of type- $(\wedge, i-1)$, for all $i \in[n]$.

Game 2.i.1. Game 2.i.1 is defined as Game 2.i.0 except that if and only if the $i$-th bit of a challenge identity is 1 , then the corresponding challenge ciphertext is semi-functional of type $(\sim, i-1)$. (Otherwise, if and only if the $i$-th bit of a challenge identity is 0 , then the corresponding challenge ciphertext is semi-functional of type $(\wedge, i-1)$, for all $j$.)

Game 2.i.2. Game 2.i.2 is defined as Game 2.i.1 except that the challenge ciphertexts are semi-functional of type $(\cdot, i)$ (where $\cdot$ can be $\wedge$ or $\sim$ as defined in Game 2.i.1, i.e., depending on the $i$-th challenge identity bit) and the user secret keys are semi-functional of type $i$. 
Table 1. Instance- $j$ challenge ciphertexts for challenge identity $i d_{j, i^{\prime}}^{*}$, for $\mathbf{g} \leftarrow$ $\operatorname{SampG}(p p)$, for $\widehat{\mathrm{g}} \leftarrow \widehat{\operatorname{SampG}}(p p, s p)$, for $\widetilde{\mathrm{g}} \leftarrow \widehat{\operatorname{SampG}}(p p, s p)$, for $R_{j, i^{\prime}} \leftarrow\{0,1\}^{k}$, and for instance- $j$ user secret keys for identity $i d$, for $\mathbf{h} \leftarrow \operatorname{SampH}(p p)$, for all $\left(j, i^{\prime}, i\right) \in[\mu] \times[q] \times[n]$. The differences between games are given by underlining.

\begin{tabular}{|c|c|}
\hline & Challenge ciphertexts for $i d_{j, i^{\prime}}^{*}$ \\
\hline Game 0 & $\operatorname{Enc}\left(m p k_{j}, i d_{j, i^{\prime}}^{*}, M_{j, i^{\prime}, b}^{*}\right)$ \\
\hline Game 1 & $\overline{\operatorname{Enc}}\left(p p, i d_{j, i^{\prime}}^{*}, M_{j, i^{\prime}, b}^{*} ; m s k_{j}, \mathbf{g} \widehat{\mathbf{g}}\right)$ \\
\hline Game 2.i.0 & $\overline{\overline{\mathrm{Enc}}}\left(p p, i d_{j, i^{\prime}}^{*}, M_{j, i^{\prime}, b}^{*} ; m s k_{j} \cdot \underline{\widehat{\mathrm{R}}_{j, i-1}}\left(\left.i d_{j, i^{\prime}}^{*}\right|_{i-1}\right), \mathbf{g} \widehat{\mathbf{g}}\right)$ \\
\hline Game 2.i.1 & $\begin{array}{l}\text { if } i d_{j, i^{\prime}, i}^{*}=0: \overline{\operatorname{Enc}}\left(p p, i d_{j, i^{\prime}}^{*}, M_{j, i^{\prime}, b}^{*} ; m s k_{j} \cdot \widehat{\mathrm{R}}_{j, i-1}\left(\left.i d_{j, i^{\prime}}^{*}\right|_{i-1}\right), \mathbf{g} \widehat{\mathbf{g}}\right) \\
\text { if } i d_{j, i^{\prime}, i}^{*}=1: \overline{\operatorname{Enc}}\left(p p, i d_{j, i^{\prime}}^{*}, M_{j, i^{\prime}, b}^{*} ; m s k_{j} \cdot \widetilde{\mathrm{R}}_{j, i-1}\left(\left.i d_{j, i^{\prime}}^{*}\right|_{i-1}\right), \underline{\mathbf{g}} \underline{\underline{\mathbf{g}}}\right)\end{array}$ \\
\hline Game 2.i.2 & if $i d_{j, i^{\prime}, i}^{*}=0: \overline{\operatorname{Enc}}\left(p p, i d_{j, i^{\prime}}^{*}, M_{j, i^{\prime}, b}^{*} ; m s k_{j} \cdot \frac{\widehat{\left.\widehat{\mathrm{R}}_{j, i}\left(\left.i d_{j, i^{\prime}}^{*}\right|_{i}\right), \mathbf{g} \widehat{\mathbf{g}}\right)}}{\widetilde{\sim}}\right.$ \\
\hline & if $i d_{j, i^{\prime}, i}^{*}=1: \overline{\operatorname{Enc}}\left(p p, i d_{j, i^{\prime}}^{*}, M_{j, i^{\prime}, b}^{*} ; m s k_{j} \cdot \overline{\widetilde{\mathrm{R}}_{j, i}\left(\left.i d_{j, i^{\prime}}^{*}\right|_{i}\right)}, \mathbf{g} \widetilde{\mathbf{g}}\right)$ \\
\hline Game 3 & 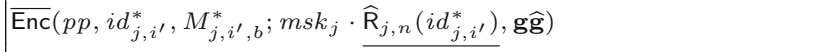 \\
\hline \multirow[t]{2}{*}{ Game 4} & $\overline{\operatorname{Enc}}\left(p p, i d_{j, i^{\prime}}^{*}, R_{j, i^{\prime}} ; m s k_{j} \cdot \widehat{\mathrm{R}}_{j, n}\left(i d_{j, i^{\prime}}^{*}\right), \mathbf{g} \widehat{\mathbf{g}}\right)$ \\
\hline & User secret keys for $i d$ \\
\hline Game 0 & $\operatorname{Ext}\left(m s k_{j}, i d\right)$ \\
\hline Game 1 & $\overline{\operatorname{Ext}}\left(p p, m s k_{j}, i d ; \mathbf{h}\right)$ \\
\hline Game 2.i.0 & $\overline{\overline{\operatorname{Ext}}}\left(p p, m s k_{j} \cdot \underline{\widehat{\mathrm{R}}_{j, i-1}}\left(\left.i d\right|_{i-1}\right) \cdot \widetilde{\mathrm{R}}_{j, i-1}\left(\left.i d\right|_{i-1}\right), i d ; \mathbf{h}\right)$ \\
\hline Game 2.i.1 & $\overline{\operatorname{Ext}}\left(p p, m s k_{j} \cdot \overline{\widehat{\mathrm{R}}_{j, i-1}\left(\left.i d\right|_{i-1}\right) \cdot \widetilde{\mathrm{R}}_{j, i-1}\left(\left.i d\right|_{i-1}\right)}, i d ; \mathbf{h}\right)$ \\
\hline Game 2.i.2 & $\overline{\operatorname{Ext}}\left(p p, m s k_{j} \cdot \widehat{\mathrm{R}}_{j, i}\left(\left.i d\right|_{i}\right) \cdot \widetilde{\mathrm{R}}_{j, i}\left(\left.i d\right|_{i}\right), i d ; \mathbf{h}\right)$ \\
\hline Game 3 & $\overline{\operatorname{Ext}}\left(p p, m s k_{j} \cdot \widehat{\widehat{\mathrm{R}}_{j, n}(i d) \cdot \widetilde{\mathrm{R}}_{j, n}(i d), i d} ; \mathbf{h}\right)$ \\
\hline Game 4 & $\overline{\operatorname{Ext}}\left(p p, m s k_{j} \cdot \widehat{\mathrm{R}}_{j, n}(i d) \cdot \widetilde{\mathrm{R}}_{j, n}(i d), i d ; \mathbf{h}\right)$ \\
\hline
\end{tabular}

Game 3. Game 3 is defined as Game 2.n.0 except that the challenge ciphertexts are semi-functional of type $(\wedge, n)$ and the user secret keys are semi-functional of type $n$.

Game 4. Game 4 is defined as Game 3 except that the challenge ciphertext messages are uniform $k$-length bitstrings, for all $\left(j, i^{\prime}\right) \in[\mu] \times[q]$.

Lemma 1 (Game 0 to Game 1). Let $\mathrm{G}$ be a group generator as defined above. If the $G$ - and $H$-subgroups property and LS1 of ENDSG hold, Game 0 and Game 1 are computationally indistinguishable. Concretely, for any PPT adversary $A$ with at most $q^{\prime}=q^{\prime}(k)$ extraction queries in each instance and running time $t$ in the $(\mu, q)-I B E-I N D-C P A$ security experiment with IBE there is an distinguisher $D$ on LS1 with running time $t^{\prime} \approx t+\mathbf{O}\left(\mu q q^{\prime} n k^{c}\right)$, for integer $n=n(k)$ and some constant $c \in \mathbb{N}$, such that

$$
\left|\operatorname{Pr}\left[S_{A, 0}\right]-\operatorname{Pr}\left[S_{A, 1}\right]\right| \leq \operatorname{Adv}_{\text {ENDSG }, \mathrm{G}, D}^{\text {ls1 }}(k, 2 n) .
$$

Proof. In Game 0, all challenge ciphertexts are normal in the sense of IBE while in Game 1, all challenge ciphertexts are pseudo-normal. In the following, we give a description and its analysis of a LS1 distinguisher that uses any efficient IBE-attacker in the $(\mu, q)$-IBE-IND-CPA sense. 
Description. The challenge input is provided as $(p p, \mathbf{T})$, where $\mathbf{T}$ is either $\mathbf{g}$ or $\mathbf{g} \widehat{\mathbf{g}}$, for $p p=\left(G, H, G_{T}, N, e, m, 2 n, \mathrm{H}\right.$, pars $), \mathbf{g} \leftarrow \operatorname{SampG}(p p)$, and $\widehat{\mathbf{g}} \leftarrow$ $\widehat{\operatorname{SampG}}(p p, s p)$. First, $D$ samples $\left(m s k_{j}\right)_{j} \leftarrow(H)^{\mu}$, sets $m p k_{j}:=\left(p p, m\left(m s k_{j}\right)\right)$, for all $j$, and sends $\left(m p k_{j}\right)_{j}$ to $A$. During the experiment, $D$ answers instance- $j$ secret key extraction queries to oracle $\operatorname{Ext}\left(m s k_{j}, \cdot\right)$, for $i d \in \mathcal{I D}$, with

$$
\overline{\operatorname{Ext}}\left(p p, m s k_{j}, i d ; \operatorname{SampH}(p p)\right),
$$

for all $j$. (We assume that $A$ queries at most $q^{\prime}=q^{\prime}(k)$ user secret keys per instance.) Then, $D$ fixes a bit $b \leftarrow\{0,1\}$. $A$ may adaptively query its Enc'-oracle; for $A$-chosen instance- $j$ challenge identities $\left(i d_{j, i}^{*}\right)_{i}$ and equal-length messages $\left(M_{j, i, 0}^{*}, M_{j, i, 1}^{*}\right)_{i}$, for all $i \in[q] . D$ returns

$$
\overline{\operatorname{Enc}}\left(p p, i d_{j, i}^{*}, M_{j, i, b}^{*} ; m s k_{j}, \mathbf{T}^{s_{j, i}}\right)
$$

to $A$, for $s_{j, i} \leftarrow \mathbb{Z}_{N}^{*}$, for all $(j, i)$. (We assume that $A$ queries at most $q$ challenge ciphertexts per instance.) Eventually, $A$ outputs a guess $b^{\prime} . D$ outputs 1 if $b^{\prime}=b$ and $A$ is valid in the sense of $(\mu, q)$-IBE-IND-CPA, else outputs 0 .

Analysis. The provided master public keys and the $A$-requested user secret keys yield the correct distribution and are consistent in the sense of Game 0 and Game 1. Due to ENDSG's $G$ - and $H$-subgroups property, we have that $\mathbf{T}$ is uniformly distributed over the generators of a nontrivial subgroup of $G^{2 n+1}$. Hence, $\mathbf{T}^{s}$, for $s \leftarrow \mathbb{Z}_{N}^{*}$, is distributed uniformly over the generators of a nontrivial subgroup of $G^{2 n+1}$ and, thus, all challenge ciphertexts yield the correct distribution in the sense of Game 0 and Game 1 . If $\mathbf{T}=\mathbf{g}$, then the challenge ciphertexts are distributed identically as in Game 0. Otherwise, i.e., if $\mathbf{T}=\mathbf{g} \widehat{\mathbf{g}}$, then the challenge ciphertexts are distributed identically as in Game 1. Hence, (2) follows.

Lemma 2 (Game 1 to Game 2.1.0). If the orthogonality property of ENDSG holds, the output distributions of Game 1 and Game 2.1.0 are the same. Concretely, for any PPT adversary $A$ in the $(\mu, q)-I B E-I N D-C P A$ security experiment with IBE defined as above it holds that

$$
\operatorname{Pr}\left[S_{A, 1}\right]=\operatorname{Pr}\left[S_{A, 2.1 .0}\right]
$$

Proof. In this bridging step, we argue that each instance- $j$ master secret key $m s k_{j}$, with $m s k_{j} \leftarrow H$, generated as in Game 1 and the (implicit) instance$j$ master secret keys $m s k_{j}^{\prime}$, with $m s k_{j}^{\prime}:=m s k_{j}^{\prime \prime} \cdot \widehat{\mathrm{R}}_{j, 0}(\varepsilon) \cdot \widetilde{\mathrm{R}}_{j, 0}(\varepsilon)$, for $m s k_{j}^{\prime \prime} \leftarrow$ $H$ and $\widehat{\mathrm{R}}_{j, 0}, \widetilde{\mathrm{R}}_{j, 0}$ defined as above, generated as in Game 2.1.0, are identically distributed, for all $j$. Note that the master public keys for $A$ contain $\left(m\left(m s k_{j}\right)\right)_{j}$; but since $\left(\left(m\left(m s k_{j}^{\prime}\right)\right)_{j}=\left(m\left(m s k_{j}^{\prime \prime}\right)\right)_{j}\right.$, which is due to the orthogonality property of ENDSG, no $\widehat{\mathrm{R}}_{j, 0}$-information and no $\widetilde{\mathrm{R}}_{j, 0}$-information is given out in the master public keys. Further, since $\left(m s k_{j}\right)_{j}$ and $\left(m s k_{j}^{\prime \prime}\right)_{j}$ are identically distributed, it follows that (3) holds. 
Lemma 3 (Game 2.i.0 to Game 2.i.1). Let G be a group generator as defined above. If the $G$ - and $H$-subgroups property and LS2 of ENDSG hold, Game 2.i.0 and Game 2.i.1 are computationally indistinguishable. Concretely, for any PPT adversary $A$ with at most $q^{\prime}=q^{\prime}(k)$ extraction queries in each instance and running time $t$ in the $(\mu, q)-I B E-I N D-C P A$ security experiment with IBE defined as above, there is a distinguisher D on LS2 with running time $t^{\prime} \approx t+\mathbf{O}\left(\mu q q^{\prime} n k^{c}\right)$, for integer $n=n(k)$ and some constant $c \in \mathbb{N}$, such that

$$
\left|\operatorname{Pr}\left[S_{2 . i .0}\right]-\operatorname{Pr}\left[S_{2 . i .1}\right]\right| \leq \operatorname{Adv}_{\mathrm{ENDSG}, \mathrm{G}, D}^{\mathrm{ls} 2}(k, 2 n),
$$

for all $i \in[n]$.

Proof. In Game 2.i.0, we have semi-functional type- $(\wedge, i-1)$ challenge ciphertexts while in Game 2.i.1, challenge ciphertexts are semi-functional of type $(\sim, i-1)$ if and only if the $i$-th challenge identity bit is 1 .

Description. The challenge input is provided as $\left(p p, \widehat{h} \widetilde{h}, \mathbf{g}^{\prime} \widehat{\mathbf{g}}^{\prime}, \mathbf{T}\right)$, where $\mathbf{T}$ is either $\mathbf{g} \widehat{\mathbf{g}}$ or $\mathbf{g} \widetilde{\mathbf{g}}$, for $p p$ as before, for $\widehat{h}, \widetilde{h}$ specified in $s p$, for $\mathbf{g}, \mathbf{g}^{\prime} \leftarrow \operatorname{SampG}(p p)$, $\widehat{\mathbf{g}}, \widehat{\mathbf{g}}^{\prime} \leftarrow \widehat{\operatorname{SampG}}(p p, s p)$, and $\widetilde{\mathbf{g}} \leftarrow \widehat{\operatorname{SampG}}(p p, s p)$. First, $D$ samples $\left(m s k_{j}\right)_{j} \leftarrow$ $(H)^{\mu}$, sets $m p k_{j}:=\left(p p, m\left(m s k_{j}\right)\right)$, for all $j$, for $m$ specified in $p p$, and sends $\left(m p k_{j}\right)_{j}$ to $A$. Further, $D$ defines a truly random function R : $[\mu] \times\{0,1\}^{i-1} \rightarrow$ $\langle\widehat{h} \widetilde{h}\rangle$. During the experiment, $D$ answers instance- $j$ secret key extraction queries to oracle $\operatorname{Ext}\left(m s k_{j}, \cdot\right)$ as

$$
\overline{\operatorname{Ext}}\left(p p, m s k_{j} \cdot \mathrm{R}\left(j,\left.i d\right|_{i-1}\right), i d ; \operatorname{SampH}(p p)\right),
$$

for $i d \in \mathcal{I D}$ and all $j$. (Again, we assume that $A$ queries at most $q^{\prime}=q^{\prime}(k)$ user secret keys per instance and we set $\left.i d\right|_{0}=\{0,1\}^{0}=$ : $\varepsilon$.) $A$ may adaptively query its Enc'-oracle; for $A$-chosen instance- $j$ challenge identity $i d_{j, i^{\prime}}^{*}=$ $i d_{j, i^{\prime}, 1}^{*} \ldots, i d_{j, i^{\prime}, n}^{*} \in \mathcal{I D}$ and equal-length messages $\left(M_{j, i^{\prime}, 0}^{*}, M_{j, i^{\prime}, 1}^{*}\right)_{i^{\prime}}$, for all $i^{\prime} \in[q]$. D returns

$$
\begin{gathered}
\overline{\operatorname{Enc}}\left(p p, i d_{j, i^{\prime}}^{*}, M_{j, i^{\prime}, b}^{*} ; m s k_{j} \cdot \mathrm{R}\left(j,\left.i d_{j, i^{\prime}}^{*}\right|_{i-1}\right),\left(\mathbf{g}^{\prime} \hat{\mathbf{g}}^{\prime}\right)^{s_{j, i^{\prime}}}\right) \quad \text { if } i d_{j, i^{\prime}, i}^{*}=0, \\
\overline{\operatorname{Enc}}\left(p p, i d_{j, i^{\prime}}^{*}, M_{j, i^{\prime}, b}^{*} ; m s k_{j} \cdot \mathrm{R}\left(j,\left.i d_{j, i^{\prime}}^{*}\right|_{i-1}\right), \mathbf{T}^{s_{j, i^{\prime}}}\right) \quad \text { if } i d_{j, i, i^{\prime}}^{*}=1,
\end{gathered}
$$

to $A$, for (fixed) $b \leftarrow\{0,1\}$, for $s_{j, i^{\prime}} \leftarrow \mathbb{Z}_{N}^{*}$, for all $\left(j, i^{\prime}\right)$. Eventually, $A$ outputs a guess $b^{\prime} . D$ outputs 1 if $b^{\prime}=b$ and $A$ is valid in the sense of $(\mu, q)$-IBE-IND-CPA, else outputs 0 .

Analysis. The master public keys yield the correct distribution as well as the requested user secret keys (which is due to ENDSG's $G$ - and $H$-subgroups property). For challenge ciphertexts, note that $\mathbf{g}^{\prime} \widehat{\mathbf{g}}^{\prime}$ and $\mathbf{T}$ are uniformly distributed over the generators of their respective nontrivial subgroup of $G^{2 n+1}$ and, hence, $\left(\mathbf{g}^{\prime} \widehat{\mathbf{g}}^{\prime}\right)^{s}$ and $\mathbf{T}^{s}$, for $s \leftarrow \mathbb{Z}_{N}^{*}$, are distributed uniformly over the generators of their respective nontrivial $G^{2 n+1}$-subgroup as well. If $\mathbf{T}=\mathbf{g} \widehat{\mathbf{g}}$, then the challenge ciphertexts are distributed identically as in Game 2.i.0. Otherwise, if $\mathbf{T}=\mathbf{g} \widetilde{\mathbf{g}}$, then the challenge ciphertexts are distributed identically as in Game 2.i.1 (where, 
in both cases, ENDSG's orthogonality and non-degeneracy properties hold; thus, $\widehat{h}$ and $\widetilde{h}$ must contain coprime nontrivial elements and the challenge ciphertexts yield the correct distribution). Hence, (4) follows.

Lemma 4 (Game 2.i.1 to Game 2.i.2). Let $\mathrm{G}$ be a group generator as defined above. If the $G$ - and $H$-subgroups property and NH of ENDSG hold, Game 2.i.1 and Game 2.i.2 are computationally indistinguishable. Concretely, for any PPT adversary $A$ with at most $q^{\prime}=q^{\prime}(k)$ extraction queries in each instance and running time $t$ in the $(\mu, q)-I B E-I N D-C P A$ security experiment with IBE defined as above there is a distinguisher $D$ on $N H$ with running time $t^{\prime} \approx t+\mathbf{O}\left(\mu q q^{\prime} n k^{c}\right)$, for integer $n=n(k)$ and some constant $c \in \mathbb{N}$, such that

$$
\left|\operatorname{Pr}\left[S_{2 . i .1}\right]-\operatorname{Pr}\left[S_{2 . i .2}\right]\right| \leq \operatorname{Adv}_{\mathrm{ENDSG}, \mathrm{G}, D}^{\mathrm{nh}}\left(k, 2 n, q^{\prime}\right),
$$

for all $i \in[n]$.

Proof. In Game 2.i.1, the challenge ciphertexts are semi-functional of type $(\wedge, i-$ $1)$ if the $i$-th bit of the challenge identity is 0 and semi-functional of type $(\sim, i-1)$ if the $i$-th bit of the challenge identity is 1, while in Game 2.i.1, all challenge ciphertexts are of type $(\cdot, i)$.

Description. The challenge input is $\left(p p, \widehat{h}, \widetilde{h}, \widehat{\mathbf{g}}_{-(2 i-1)}, \widetilde{\mathbf{g}}_{-2 i},\left(\mathbf{T}_{1}, \ldots, \mathbf{T}_{q^{\prime}}\right)\right)$, where $\mathbf{T}_{i^{\prime}}$ equals either

$$
\left(h_{i^{\prime}, 0}, \ldots, h_{i^{\prime}, n}\right) \quad \text { or } \quad\left(h_{i^{\prime}, 0}, \ldots, h_{i^{\prime}, 2 i-1} \cdot(\widehat{h})^{\widehat{\gamma}_{j, i^{\prime}}}, h_{i^{\prime}, 2 i} \cdot(\widetilde{h})^{\widetilde{\gamma}_{j, i^{\prime}}}, \ldots, h_{i^{\prime}, n}\right),
$$

for $p p$ as before, $\widehat{h}, \widetilde{h}$ as in $s p$, for $\widehat{\mathbf{g}} \leftarrow \widehat{\operatorname{SampG}}(p p, s p)$, for $\widetilde{\mathbf{g}} \leftarrow \widehat{\operatorname{SampG}}(p p, s p)$, for $\left(h_{i^{\prime}, 0}, \ldots, h_{i^{\prime}, n}\right) \leftarrow \operatorname{SampH}(p p)$, for uniform $\widehat{\gamma}_{j, i^{\prime}}, \widetilde{\gamma}_{j, i^{\prime}} \in \mathbb{Z}_{\operatorname{ord}(H)}^{*} . D$ samples $\left(m s k_{j}\right)_{j} \leftarrow(H)^{\mu}$, sets $m p k_{j}:=\left(p p, m\left(m s k_{j}\right)\right)$, for all $j$, for $m$ specified in $p p$, and sends $\left(m p k_{j}\right)_{j}$ to $A$. Further, $D$ defines random functions $\widehat{\mathrm{R}}_{j, i-1}, \widetilde{\mathrm{R}}_{j, i-1}$ as above. In addition, for identity $i d=i d_{1} \ldots i d_{n} \in \mathcal{I D}$, we define

$$
\widehat{\mathrm{R}}_{j, i}\left(\left.i d\right|_{i}\right):=\widehat{\mathrm{R}}_{j, i-1}\left(\left.i d\right|_{i-1}\right) \text { and (implicitly) } \widetilde{\mathrm{R}}_{j, i}\left(\left.i d\right|_{i}\right):=\widetilde{\mathrm{R}}_{j, i-1}\left(\left.i d\right|_{i-1}\right) \cdot(\widetilde{h})^{\widetilde{\gamma}_{j, i^{\prime}}}
$$

if $i d_{i}=0$ and

$$
\widetilde{\mathrm{R}}_{j, i}\left(\left.i d\right|_{i}\right):=\widetilde{\mathrm{R}}_{j, i-1}\left(\left.i d\right|_{i-1}\right) \text { and (implicitly) } \widehat{\mathrm{R}}_{j, i}\left(\left.i d\right|_{i}\right):=\widehat{\mathrm{R}}_{j, i-1}\left(\left.i d\right|_{i-1}\right) \cdot(\widehat{h})^{\widehat{\gamma}_{j, i^{\prime}}}
$$

if $i d_{i}=1$. During the experiment, $D$ returns the $i_{j}$-th instance- $j$ secret-keyextraction query to oracle $\operatorname{Ext}\left(m s k_{j}, \cdot\right)$ for an identity $i d$, with prefix $\left.i d\right|_{i}$ not an prefix of an already queried identity, as

$$
\begin{array}{ll}
\overline{\operatorname{Ext}}\left(p p, m s k_{j} \cdot \widehat{\mathrm{R}}_{j, i}\left(\left.i d\right|_{i}\right) \cdot \widetilde{\mathrm{R}}_{j, i-1}\left(\left.i d\right|_{i-1}\right), i d ; \mathbf{T}_{i^{\prime}}\right) & \text { if } i d_{i}=0, \\
\overline{\operatorname{Ext}}\left(p p, m s k_{j} \cdot \widehat{\mathrm{R}}_{j, i-1}\left(\left.i d\right|_{i-1}\right) \cdot \widetilde{\mathrm{R}}_{j, i}\left(\left.i d\right|_{i}\right), i d ; \mathbf{T}_{i^{\prime}}\right) & \text { if } i d_{i}=1 .
\end{array}
$$

(We assume that $A$ queries at most $q^{\prime}=q^{\prime}(k)$ user secret keys per instance.) For an identity prefixes $\left.i d\right|_{i}$ that is an prefix of an already queried identity, let $i^{\prime \prime} \in\left[q^{\prime}\right]$ be the index of that query. In that case, $D$ returns

$$
\begin{array}{ll}
\overline{\operatorname{Ext}}\left(p p, m s k_{j} \cdot \widehat{\mathrm{R}}_{j, i}\left(\left.i d\right|_{i}\right) \cdot \widetilde{\mathrm{R}}_{j, i-1}\left(\left.i d\right|_{i-1}\right), i d ; \mathbf{T}_{i^{\prime \prime}} \cdot \operatorname{SampH}(p p)\right) & \text { if } i d_{i}=0, \\
\overline{\operatorname{Ext}}\left(p p, m s k_{j} \cdot \widehat{\mathrm{R}}_{j, i-1}\left(\left.i d\right|_{i-1}\right) \cdot \widetilde{\mathrm{R}}_{j, i}\left(\left.i d\right|_{i}\right), i d ; \mathbf{T}_{i^{\prime \prime}} \cdot \operatorname{SampH}(p p)\right) & \text { if } i d_{i}=1,
\end{array}
$$


for all $i^{\prime \prime}$. (Note that we use SampH to re-randomize the $H^{2 n+1}$-subgroup elements of $\mathbf{T}_{i^{\prime \prime}}$.) Further, $A$ may adaptively query its Enc'-oracle; for $A$-chosen instance- $j$ challenge identity $i d_{j, i^{\prime \prime \prime}}^{*}=i d_{j, i^{\prime \prime \prime}, 1}^{*} \ldots, i d_{j, i^{\prime \prime \prime}, n}^{*}$ and equal-length messages $\left(M_{j, i^{\prime \prime \prime}, 0}^{*}, M_{j, i^{\prime \prime \prime}, 1}^{*}\right)_{i^{\prime \prime \prime}}$, for all $i^{\prime \prime \prime}$. $D$ returns

$$
\overline{\operatorname{Enc}}\left(p p, i d_{j, i^{\prime \prime \prime}}^{*}, M_{j, i^{\prime \prime \prime}, b}^{*} ; m s k_{j} \cdot \widehat{\mathrm{R}}_{j, i}\left(\left.i d_{j, i^{\prime \prime \prime}}^{*}\right|_{i}\right),\left(\mathbf{g}_{-(2 i-1)} \widehat{\mathbf{g}}_{-(2 i-1)}\right)^{s_{j, i^{\prime \prime \prime}}}\right) \text { if } i d_{j, i^{\prime \prime \prime}, i}^{*}=0,
$$

$$
\overline{\operatorname{Enc}}\left(p p, i d_{j, i^{\prime \prime \prime}}^{*}, M_{j, i^{\prime \prime \prime}, b}^{*} ; m s k_{j} \cdot \widetilde{\mathrm{R}}_{j, i}\left(\left.i d_{j, i^{\prime \prime \prime}}^{*}\right|_{i}\right),\left(\mathbf{g}_{-2 i} \widetilde{\mathbf{g}}_{-2 i}\right)^{s_{j, i^{\prime \prime \prime}}}\right) \text { if } i d_{j, i^{\prime \prime \prime}, i}^{*}=1,
$$

to $A$, for $s_{j, i^{\prime \prime \prime}} \leftarrow \mathbb{Z}_{N}^{*}$, for $\mathbf{g} \leftarrow \operatorname{SampG}(p p)$, for fixed $b \leftarrow\{0,1\}$, for all $\left(j, i^{\prime \prime \prime}\right)$. (Note that a modified Enc-input is provided which has only $4 n+2$ elements instead of $4 n+4$ elements. Nevertheless, the removed elements are not needed to generate a valid ciphertext since it is consistent with $i d_{j, i^{\prime \prime \prime}}^{*}$; thus, we assume that the algorithm works as defined above.) Eventually, $A$ outputs a guess $b^{\prime}$. $D$ outputs 1 if $b^{\prime}=b$ and $A$ is valid in the sense of $(\mu, q)$-IBE-IND-CPA, else outputs 0 .

Analysis. Note that the provided master public keys yield the correct distribution; for the $A$-requested user secret keys note that, since $\widehat{h}$ and $\widetilde{h}$ have nontrivial $H$-elements of coprime order (again, this is due to the orthogonality and nondegeneracy properties), the random functions $\widehat{\mathrm{R}}_{j, i-1}, \widehat{\mathrm{R}}_{j, i}$ and $\widetilde{\mathrm{R}}_{j, i-1}, \widetilde{\mathrm{R}}_{j, i}$ yield the correct distributions in the sense of Game 2.i.1 and Game 2.i.2, respectively. Due to the $G$ - and $H$-subgroups property of ENDSG, $\mathbf{g}_{-(2 i-1)}$ and $\widehat{\mathbf{g}}_{-(2 i-1)}$ as well as $\mathbf{g}_{-2 i}$ and $\widetilde{\mathbf{g}}_{-2 i}$ are uniformly distributed over the generators of their respective nontrivial subgroups of $G^{2 n}$ and, thus, $\left(\mathbf{g}_{-(2 i-1)} \widehat{\mathbf{g}}_{-(2 i-1)}\right)^{s}$ and $\left(\mathbf{g}_{-2 i} \widetilde{\mathbf{g}}_{-2 i}\right)^{s}$, for $s \leftarrow \mathbb{Z}_{N}^{*}$, are distributed uniformly over the generators of their respective nontrivial subgroup of $G^{2 n}$. Further, if $i d_{j, i^{\prime \prime \prime}, i}^{*}=0$, then it holds that $\widehat{\mathrm{R}}_{j, i}\left(\left.i d_{j, i^{\prime \prime \prime}}^{*}\right|_{i}\right)=\widehat{\mathrm{R}}_{j, i}\left(\left.i d_{j, i^{\prime \prime \prime}}^{*}\right|_{i-1}\right)$ and all required semi-functional components $\widehat{\mathbf{g}}_{-(2 i-1)}$ to create the challenge ciphertexts are given. Analogously, if $i d_{j, i^{\prime \prime \prime}, i}^{*}=1$, then we have $\widetilde{\mathrm{R}}_{j, i}\left(\left.i d_{j, i^{\prime \prime \prime}}^{*}\right|_{i}\right)=\widetilde{\mathrm{R}}_{j, i-1}\left(\left.i d_{j, i^{\prime \prime \prime}}^{*}\right|_{i-1}\right)$ and all necessary semi-functional components $\widetilde{\mathbf{g}}_{-2 i}$ are provided as needed. (Thus, the challenge ciphertexts and user secret keys yield the correct distribution.) If $\mathbf{T}_{i^{\prime}}=\left(h_{i^{\prime}, 0}, \ldots, h_{i^{\prime}, n}\right)$, for all $i^{\prime}$, then the user secret keys are distributed identically as in Game 2.i.1. If

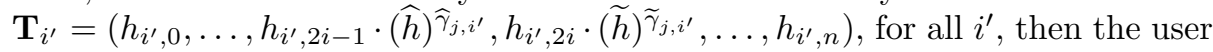
secret keys are distributed identically as in Game 2.i.2. Thus, (5) follows.

Lemma 5 (Game 2.i-1.2 to Game 2.i.0). Let G be a group generator as defined above. If the $G$ - and $H$-subgroups property and LS2 of ENDSG hold, Game 2.i-1.1 and Game 2.i.0 are computationally indistinguishable. Concretely, for any PPT adversary $A$ with at most $q^{\prime}=q^{\prime}(k)$ extraction queries in each instance and running time $t$ in the $(\mu, q)-I B E-I N D-C P A$ security experiment with IBE, defined as above, there is a distinguisher $D$ with running time $t^{\prime} \approx$ $t+\mathbf{O}\left(\mu q q^{\prime} n k^{c}\right)$, for integer $n=n(k)$ and some constant $c \in \mathbb{N}$, such that

$$
\left|\operatorname{Pr}\left[S_{2 . i-1.2}\right]-\operatorname{Pr}\left[S_{2 . i .0}\right]\right| \leq \operatorname{Adv}_{\mathrm{ENDSG}, \mathrm{G}, D}^{\mathrm{ls} 2}(k, 2 n),
$$

for all $i \in[n] \backslash\{1\}$. 
Proof. The proof is essentially the proof of Lemma 3 except that the challenge ciphertexts and user secret keys depend on the $(i-1)$-th instead of the $i$-th challenge identity bit.

Lemma 6 (Game 2.n.2 to Game 3). Let G be a group generator as defined above. If the $G$ - and $H$-subgroups property and LS2 of ENDSG hold, Game 2.n.2 and Game 3 are computationally indistinguishable. Concretely, for any PPT adversary $A$ with at most $q^{\prime}=q^{\prime}(k)$ extraction queries in each instance and running time $t$ in the $(\mu, q)-I B E-I N D-C P A$ security experiment with IBE defined as above there is a distinguisher $D$ with running time $t^{\prime} \approx t+\mathbf{O}\left(\mu q q^{\prime} n k^{c}\right)$, for integer $n=n(k)$ and some constant $c \in \mathbb{N}$, such that

$$
\left|\operatorname{Pr}\left[S_{A, 2 . n .2}\right]-\operatorname{Pr}\left[S_{A, 3}\right]\right| \leq \operatorname{Adv}_{\mathrm{ENDSG}, \mathrm{G}, D}^{\mathrm{ls} 2}(k, 2 n) .
$$

Proof. It is easy to see that Game 3 and a potential Game $2 . n+1.0$ would be identical. Thus, we can reassemble the proof of Lemma 5 with $i:=n+1$ and (7) directly follows.

Lemma 7 (Game 3 to Game 4). Then Game 3 and Game 4 are statistically indistinguishable. Concretely, for any PPT adversary $A$ on the $(\mu, q)-I B E-I N D$ CPA security of IBE defined as above it holds that

$$
\left|\operatorname{Pr}\left[S_{A, 3}\right]-\operatorname{Pr}\left[S_{A, 4}\right]\right| \leq \mu q \cdot \mathbf{O}\left(2^{-k}\right) .
$$

Proof. In Game 4, we replace each challenge message $M_{j, i^{\prime}, b}$, for challenge bit $b \in\{0,1\}$, with a (fresh) uniformly random $k$-length bitstring $R_{j, i^{\prime}} \leftarrow\{0,1\}^{k}$. We argue with ENDSG's non-degeneracy property and the universality of $\mathrm{H}$ for this change. Concretely, for instance- $j$ Game-3 challenge ciphertexts

$$
\begin{aligned}
& \overline{\operatorname{Enc}}\left(p p, i d_{j, i^{\prime}}^{*}, M_{j, i^{\prime}, b}^{*} ; m s k_{j} \cdot \widehat{\mathrm{R}}_{j, n}\left(i d_{j, i^{\prime}}^{*}\right),(\mathbf{g} \widehat{\mathbf{g}})^{s_{j, i^{\prime}}}\right) \\
& =\left(\left(g_{0} \widehat{g}_{0}\right)^{s_{j, i^{\prime}}},\left(\prod_{i=1}^{n} g_{2 i-i d_{j, i^{\prime}, i}^{*}} \widehat{g}_{2 i-i d_{j, i^{\prime}, i}^{*}}\right)^{s_{j, i^{\prime}}}, \mathrm{H}\left(e\left(\left(g_{0} \widehat{g}_{0}\right)^{s_{j, i^{\prime}}}, m s k_{j} \cdot \widehat{\mathrm{R}}_{j, n}\left(i d_{j, i^{\prime}}^{*}\right)\right)\right)\right. \\
& \left.\quad \oplus M_{j, i^{\prime}, b}^{*}\right),
\end{aligned}
$$

for $\mathbf{g} \leftarrow \operatorname{SampG}(p p)$, for $\widehat{\mathbf{g}} \leftarrow \widehat{\operatorname{SampG}}(p p, s p)$, for $s_{j, i^{\prime}} \leftarrow \mathbb{Z}_{N}^{*}$, for all $i^{\prime} \in[q]$, note

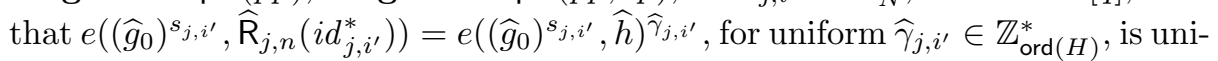
formly distributed in a subgroup $G_{T}^{\prime} \subset G_{T}$ due to the non-degeneracy property of ENDSG. Furthermore, since $A$ is a weak adversary, all the $\widehat{\mathrm{R}} j, n$ are for different preimages and thus independently random. Hence, since $\mathrm{H}$ is a (randomly chosen) universal hash function, we have that $\varepsilon:=\mathrm{SD}((\mathrm{H}, \mathrm{H}(X)) ;(\mathrm{H}, U))=$ $\mathbf{O}\left(2^{-k}\right)$, for $X \leftarrow G_{T}^{\prime}$ and $U \leftarrow\{0,1\}^{k}$. A union bound yields (8).

Lemma 8 (Game 4). For any PPT adversary $A$ in the $(\mu, q)-I B E-I N D-C P A$ security experiment with IBE defined as above it holds that

$$
\operatorname{Pr}\left[S_{A, 4}\right]=1 / 2 .
$$


Proof. In Game 4, for (uniform) challenge bit $b \in\{0,1\}$, we provide the adversary $A$ with challenge ciphertexts that include only a uniform $k$-length bitstring instead of a $A$-chosen $b$-dependent messages, for each instance and challenge. Hence, $b$ is completely hidden from $A$ and (9) follows.

Taking (2), (3), (4), (5), (6), (7), (8), and (9) together, shows (1).

From Weak to Full $(\mu, q)$-IBE-IND-CPA Security. The analysis above shows only weak security: we must assume that the adversary $A$ never asks for encryptions under the same challenge identity and for the same scheme instance twice. We do not know how to remove this restriction assuming only the abstract properties of ENDSGs. However, at the cost of one tight additional reduction to (a slight variant of) the Bilinear Decisional Diffie-Hellman (BDDH) assumption, we can show full $(\mu, q)$-IBE-IND-CPA security.

Concretely, in Game 3, challenge ciphertexts for $A$ are prepared using (the hash value of) $e\left(\widehat{g}_{0}^{s}, \widehat{h}^{\gamma}\right)$ as a mask to hide the plaintext behind. Here, $\widehat{g}_{0}^{s}$ and $\widehat{h}$ are public (as part of the ciphertext, resp. public parameters), $s$ is a fresh exponent chosen randomly for each encryption, and $\gamma$ is a random exponent that however only depends on the scheme instance and identity. (Thus, $\gamma$ will be reused for different encryptions under the same identity). Hence, if we show that many tuples $\left(\widehat{g}^{s_{i}}, e\left(\widehat{g}_{0}^{s_{i}}, \widehat{h}^{\gamma}\right)\right)$ (for different $s_{i}$ but the same $\gamma$ ) are computationally indistinguishable from random tuples, we obtain that even multiple encryptions under the same identity hide the plaintexts, and we obtain full security.

Of course, the corresponding reduction should be tight, in the sense that it should not degrade in the number of tuples, or in the number of considered $\gamma$. In the full version, we show such a reduction to the BDDH assumption (in suitable subgroups of $G$ ). (In a nutshell, we set up $e\left(\widehat{g}_{0}^{s}, \widehat{h}^{\gamma}\right)=e(g, g)^{a b c}$ for a generator $g$ and random exponents $a, b, c$ with $\widehat{g}_{0}^{s}=g^{a}$ and $\widehat{h}^{\gamma}=g^{b c}$. The BDDH assumption now states that $e(g, g)^{a b c}$ looks random even given $g, g^{a}, g^{b}, g^{c}$. Furthermore, by the random self-reducibility of $\mathrm{BDDH}$, the corresponding reduction is tight.)

\section{Instantiations of ENDSGs in Composite-Order Groups}

Assumptions in Groups with Composite Order. We slightly modify two (known) dual system assumptions (i.e., see DS1, DS3 below, and [11]) and define one (new) dual system assumption (see DS2 below). Let $\mathrm{G}(k, 4)$ be a compositeorder group generator that outputs the following group parameters $(G, H=$ $\left.G, G_{T}, N, e, g, g_{p_{1}}, g_{p_{2}}, g_{p_{3}}, g_{p_{4}}\right)$ with the composite-order groups $G, G_{T}$, each of order $N=p_{1} \cdots p_{4}$, for pairwise-distinct $k$-bit primes $\left(p_{i}\right)_{i}$. Further, $g_{p_{i}}$ is a generator of the subgroup $G_{p_{i}} \subset G$ of order $p_{i}$, and $g$ is a generator of $G$. More generally, we write $G_{q} \subseteq G$ for the unique subgroups of order $q$. The assumptions in groups with composite order are as follows:

Dual System Assumption 1 (DS1). For any PPT adversary $D$, the function

$$
\operatorname{Adv}_{\mathrm{G}, D}^{\mathrm{ds} 1}(k):=\mid \operatorname{Pr}\left[D\left(\text { pars }, g_{p_{1}}^{\prime}\right)=1\right]-\operatorname{Pr}\left[D\left(\text { pars }, g_{p_{1} p_{2}}^{\prime}\right)=1\right] \mid
$$


is negligible in $k$, for $\left(G, G_{T}, N, e, g,\left(g_{p_{i}}\right)_{i}\right) \leftarrow \mathrm{G}(k, 4)$,

$$
\text { pars }:=\left(G, G_{T}, N, e, g, g_{p_{1}}, g_{p_{3}}, g_{p_{4}}\right) \text {, and } g_{p_{1}}^{\prime} \stackrel{g}{\leftarrow} G_{p_{1}}, g_{p_{1} p_{2}}^{\prime} \stackrel{g}{\leftarrow} G_{p_{1} p_{2}} \text {. }
$$

Dual System Assumption 2 (DS2). For any PPT adversary $D$, the function

$$
\operatorname{Adv}_{\mathrm{G}, D}^{\mathrm{ds} 2}(k):=\mid \operatorname{Pr}\left[D\left(\text { pars }, g_{p_{1} p_{2}}^{\prime}\right)=1\right]-\operatorname{Pr}\left[D\left(\text { pars }, g_{p_{1} p_{3}}^{\prime}\right)=1\right] \mid
$$

is negligible in $k$, for $\left(G, G_{T}, N, e, g,\left(g_{p_{i}}\right)_{i}\right) \leftarrow \mathrm{G}(k, 4)$,

$$
\begin{gathered}
\text { pars }:=\left(G, G_{T}, N, e, g, g_{p_{1}}, g_{p_{4}}, g_{p_{1} p_{2}}, g_{p_{2} p_{3}}\right), \\
g_{p_{1} p_{2}} \stackrel{g}{\longleftarrow} G_{p_{1} p_{2}}, g_{p_{2} p_{3}} \stackrel{g}{\longleftarrow} G_{p_{2} p_{3}} \text {, and } g_{p_{1} p_{2}}^{\prime} \stackrel{g}{\longleftarrow} G_{p_{1} p_{2}}, g_{p_{1} p_{3}}^{\prime} \stackrel{g}{\longleftarrow} G_{p_{1} p_{3}} .
\end{gathered}
$$

Dual System Assumption 3 (DS3). For any PPT adversary $D$, the function

$$
\operatorname{Adv}_{\mathrm{G}, D}^{\mathrm{ds} 3}(k):=\mid \operatorname{Pr}\left[D\left(\text { pars }, g_{p_{2}}^{x y}, g_{p_{3}}^{x y}\right)=1\right]-\operatorname{Pr}\left[D\left(\text { pars }, g_{p_{2}}^{x y+\gamma^{\prime}}, g_{p_{3}}^{x y+\gamma^{\prime}}\right)=1\right] \mid
$$

is negligible in $k$, for $\left(G, G_{T}, N, e, g,\left(g_{p_{i}}\right)_{i}\right) \leftarrow \mathrm{G}(k, 4)$,

$$
\begin{aligned}
\text { pars }:= & \left(G, G_{T}, N, e, g, g_{p_{1}}, g_{p_{2}}, g_{p_{3}}, g_{p_{4}}, g_{p_{2}}^{x} \widehat{X}_{4}, g_{p_{2}}^{y} \widehat{Y}_{4}, g_{p_{3}}^{x} \widetilde{X}_{4}, g_{p_{3}}^{y} \widetilde{Y}_{4}\right), \\
& \widehat{X}_{4}, \widetilde{X}_{4}, \widehat{Y}_{4}, \widetilde{Y}_{4} \stackrel{g}{\leftarrow} G_{p_{4}}, x, y, \leftarrow \mathbb{Z}_{N}^{*}, \text { and } \gamma^{\prime} \leftarrow \mathbb{Z}_{N}^{*} .
\end{aligned}
$$

ENDSGs in Groups with Composite Order. Let $\mathrm{G}(k, 4)$ be as defined above. For simplicity, we write $g_{i}:=g_{p_{i}}$ and $g_{i j}:=g_{p_{i} p_{j}}$, for all $(i, j) \in[4] \times[4]$. We instantiate ENDSGs ENDSG Eo $=($ SampP, SampG, SampH, SampG,$\widehat{\operatorname{SampG}})$ in composite-order groups as follows:

Parameter Sampling. SampP $(k, n)$, given $k$ and $n$, samples $\left(G, H, G_{T},\left(p_{i}\right)_{i}, e\right.$, $\left.g, h,\left(g_{i}\right)_{i}\right) \leftarrow \mathrm{G}(k, 4)$ and outputs $p p:=\left(G, H, G_{T}, N, g, e, m, n\right.$, pars, $\left.\mathrm{H}\right)$ and $s p:=(\widehat{h}, \widetilde{h}, \widehat{p a r s}, \widehat{p a r s})$, for

- $m: H \rightarrow G_{T}, h^{\prime} \mapsto e\left(g_{1}, h^{\prime}\right)$,

- $\operatorname{pars}:=\left(g_{1}, g_{4}, g_{1}^{\mathbf{w}}, h, h^{\mathbf{w}} \cdot \mathbf{R}_{4}\right)$, for $\mathbf{w} \leftarrow\left(\mathbb{Z}_{N}^{*}\right)^{n}, \mathbf{R}_{4} \stackrel{g}{\leftarrow}\left(G_{p_{4}}\right)^{n}$,

- $\widehat{h} \stackrel{g}{\leftarrow} G_{p_{2} p_{4}}, \widetilde{h} \stackrel{g}{\longleftarrow} G_{p_{3} p_{4}}$,

- $\widehat{\text { pars } s}:=\left(g_{2}, g_{2}^{\mathbf{w}}\right)$, pars $:=\left(g_{3}, g_{3}^{\mathbf{w}}\right)$.

$G$-Group Sampling. SampG $(p p)$ samples $s \leftarrow \mathbb{Z}_{N}^{*}$ and outputs $\left(g_{1}^{s}, g_{1}^{s \cdot \mathbf{w}}\right)$.

$H$-Group Sampling. SampH $(p p)$ samples $r \leftarrow \mathbb{Z}_{N}^{*}$ and outputs $\left(h^{r}, h^{r \cdot \mathbf{w}} \cdot \mathbf{R}^{\prime}{ }_{4}\right)$, for $\mathbf{R}_{4}^{\prime} \stackrel{g}{\leftarrow}\left(G_{p_{4}}\right)^{n}$.

Semi-functional $G$-group Sampling 1. $\widehat{\operatorname{SampG}}(p p, s p)$ samples $s \leftarrow \mathbb{Z}_{N}^{*}$ and outputs $\left(g_{2}^{s}, g_{2}^{s \cdot \mathbf{w}}\right)$.

Semi-functional $G$-group Sampling 2. $\widetilde{\operatorname{SampG}}(p p, s p)$ samples $s \leftarrow \mathbb{Z}_{N}^{*}$ and outputs $\left(g_{3}^{s}, g_{3}^{s \cdot \mathbf{w}}\right)$.

Correctness of ENDSG $\mathrm{co}_{\mathrm{co}}$. For all $k, n \in \mathbb{N}$ and group parameters $\left(G, H, G_{T}, N\right.$, $\left.e, g, h,\left(g_{i}\right)_{i}\right) \leftarrow \mathrm{G}(k, 4)$, we have: 
Associativity. For all $s, r \leftarrow \mathbb{Z}_{N}^{*}$, for all $\left(g_{1}^{s}, g_{1}^{s \cdot \mathbf{w}}\right) \leftarrow \operatorname{SampG}(p p ; s)$, for all $\left(h^{r}, h^{r \cdot \mathbf{w}} \cdot \mathbf{R}_{4}^{\prime}\right) \leftarrow \operatorname{SampH}(p p ; r)$, for $\mathbf{R}_{4}^{\prime}=\left(R_{i}^{\prime}\right)_{i} \in\left(G_{p_{4}}\right)^{n}$, it holds that

$$
e\left(g_{1}^{s}, h^{r \cdot w_{i}} \cdot R_{i}^{\prime}\right)=e\left(g_{1}^{s}, h^{r \cdot w_{i}}\right)=e\left(g_{1}^{s \cdot w_{i}}, h^{r}\right)
$$

for all $i \in[n]$, and for $\mathbf{w}=\left(w_{1}, \ldots, w_{n}\right) \in\left(\mathbb{Z}_{N}^{*}\right)^{n}$.

Projective. For all $s \leftarrow \mathbb{Z}_{N}^{*}$, for all $h^{\prime} \in H$, it holds that $m\left(h^{\prime}\right)^{s}=e\left(g_{1}, h^{\prime}\right)^{s}=$ $e\left(g_{1}^{s}, h^{\prime}\right)$. (Note that $g_{1}^{s}$ is the first output of $\operatorname{SampG}(p p ; s)$.)

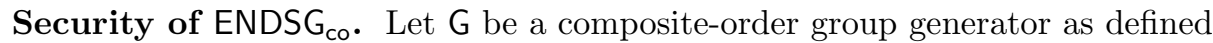
above, for all $k, n, \in \mathbb{N}$, for all $(p p, s p) \leftarrow \operatorname{SampP}(k, n)$, we have:

Orthogonality. For $\widehat{h}, \widetilde{h}$ specified in $s p$, we have

$$
m(\widehat{h})=e\left(g_{1}, \widehat{h}\right)=e\left(\left(g^{p_{2} p_{3} p_{4}}\right)^{\gamma_{g_{1}}},\left(g^{p_{1} p_{3}}\right)^{\gamma_{\widehat{h}}}\right)=1
$$

and

$$
m(\widetilde{h})=e\left(g_{1}, \widetilde{h}\right)=e\left(\left(g^{p_{2} p_{3} p_{4}}\right)^{\gamma_{g_{1}}},\left(g^{p_{1} p_{2}}\right)^{\gamma_{\tilde{h}}}\right)=1
$$

for suitable exponents $\gamma_{g_{1}}, \gamma_{\widehat{h}}, \gamma_{\widetilde{h}} \in \mathbb{Z}_{N}^{*}$. Further, for $g_{1}^{s}, g_{2}^{s^{\prime}}$, and $g_{3}^{s^{\prime \prime}}$ that are the first outputs of SampG $(p p ; s) \widehat{\operatorname{SampG}}\left(p p, s p ; s^{\prime}\right)$, and $\widehat{\operatorname{SampG}}\left(p p, s p ; s^{\prime \prime}\right)$, for $s, s^{\prime}, s^{\prime \prime} \leftarrow \mathbb{Z}_{N}^{*}$, we have $e\left(g_{1}^{s}, \widehat{h}\right)=e\left(g_{1}^{s}, \widetilde{h}\right)=e\left(g_{2}^{s^{\prime}}, \widetilde{h}\right)=e\left(g_{3}^{s^{\prime \prime}}, \widehat{h}\right)=1$.

$G$ - and $H$-subgroups. Since $g_{1}, g_{2}$, and $g_{3}$ are generators of subgroups $G_{p_{1}}$, $G_{p_{2}}$, and $G_{p_{3}}$ of coprime order, the outputs of SampG, $\widehat{\operatorname{SampG}}$, and $\widehat{\text { SampG }}$ are uniform over the generators, which generates nontrivial subgroups of $G$ of coprime order. Since $h$ is a generator of $H$ and $\mathbf{R}_{4}^{\prime}$ is uniform over the generators of $\left(G_{p_{4}}\right)^{n}$, the output of SampH is uniformly distributed over the generators of $H$.

Non-degeneracy. For the first output $g_{2}^{s}$ of $\widehat{\operatorname{SampG}}(p p, s p ; s)$ (with uniform $\left.s \in \mathbb{Z}_{N}^{*}\right)$, and for $\widehat{h} \in G_{p_{2} p_{3}}$ as specified in $s p$, it holds that $e\left(g_{2}^{s}, \widehat{h}\right)=e\left(g_{2}, \widehat{h}\right)^{s}$ is uniformly distributed over the generators of the subgroup generated by $e\left(g_{2}, \widehat{h}\right)$. Similarly, for the first output $g_{3}^{s}$ of $\widehat{\operatorname{SampG}}(p p, s p ; s)$, it holds that $e\left(g_{3}^{s}, \widetilde{h}\right)=e\left(g_{3}, \widetilde{h}\right)^{s}$ is distributed uniformly over the generators of the subgroup generated by $e\left(g_{3}, \widetilde{h}\right)$.

Left-Subgroup Indistinguishability 1. We prove the following lemma

Lemma 9 (DS1 to LS1). For any PPT adversary $D$ with running time $t$ on LS1 of ENDSG $\mathrm{Eo}_{\mathrm{co}}$ as defined above there is a distinguisher $D^{\prime}$ on DS1 with running time $t^{\prime} \approx t$ such that

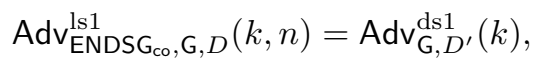

for $\mathrm{G}$ as defined above. Hence, LS1 holds under DS1.

Proof. Description. The challenge input to $D^{\prime}$ is provided as (pars, $\mathbf{T}$ ), where $\mathbf{T}$ is either $g_{1}^{\prime} \leftarrow G_{p_{1}}$ or $g_{12}^{\prime} \leftarrow G_{p_{1} p_{2}}$, for pars $=\left(G, G_{T}, N, e, g, g_{1}, g_{3}\right.$, $\left.g_{4}\right)$. First, $D^{\prime}$ sets the public parameter as $p p:=\left(G, H:=G, G_{T}, N, g, e, m\right.$, 
$n$, pars $\left.^{\prime}\right)$, for $m: h^{\prime} \mapsto e\left(g_{1}, h^{\prime}\right)$, pars $^{\prime}:=\left(g_{1}, g_{4}, g_{1}^{\mathbf{w}}, h:=g, h^{\mathbf{w}}\right)$, for $\mathbf{w} \leftarrow$ $\left(\mathbb{Z}_{N}^{*}\right)^{n}$, and for some integer $n$ determined by $D^{\prime}$. Then, $D^{\prime}$ sends $\left(p p, \mathbf{T}, \mathbf{T}^{\mathbf{w}}\right)$ to $D$. Finally, $D$ outputs a value which $D^{\prime}$ forwards to its own challenger.

Analysis. Note that $p p$ is distributed as defined in LS1. If $\mathbf{T}=g_{1}^{\prime}$, then $\left(g_{1}^{\prime},\left(g_{1}^{\prime}\right)^{\mathbf{w}}\right)$ is distributed as the output of SampG $(p p)$ as needed and, hence, $\operatorname{Pr}\left[D^{\prime}\left(\right.\right.$ pars,$\left.\left.g_{1}^{\prime}\right)=1\right]=\operatorname{Pr}\left[D\left(p p,\left(g_{1}^{\prime},\left(g_{1}^{\prime}\right)^{\mathbf{w}}\right)\right)=1\right]$ follows. Otherwise, if $\mathbf{T}=g_{12}^{\prime}$, then $\left(g_{12}^{\prime},\left(g_{12}^{\prime}\right)^{\mathbf{w}}\right)$ is distributed as SampG $(p p) \cdot \widehat{\operatorname{SampG}}(p p, s p)$, for suitable $s p$, as desired and, hence, we have that $\operatorname{Pr}\left[D^{\prime}\left(\right.\right.$ pars, $\left.\left.g_{12}^{\prime}\right)=1\right]=$ $\operatorname{Pr}\left[D\left(p p,\left(g_{12}^{\prime},\left(g_{12}^{\prime}\right)^{\mathbf{w}}\right)\right)=1\right]$. As a consequence, (10) follows.

Left-subgroup indistinguishability 2. We prove the following lemma

Lemma 10 (DS2 to LS2). For any PPT adversary D with running time

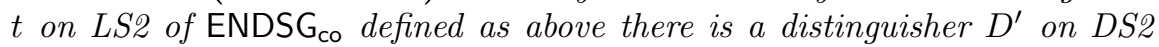
with running time $t^{\prime} \approx t$ such that

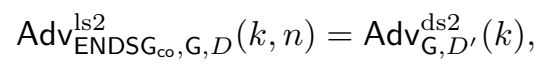

for $\mathrm{G}$ as defined above. Hence, LS2 holds under DS2.

Proof. Description. The challenge input to $D^{\prime}$ is provided as (pars, $\mathbf{T}$ ), where $\mathbf{T}$ is either $g_{12}^{\prime} \leftarrow G_{p_{1} p_{2}}$ or $g_{13}^{\prime} \leftarrow G_{p_{1} p_{3}}$, for pars $=\left(G, G_{T}, N, e, g, g_{1}\right.$, $\left.g_{4}, g_{12}, g_{23}\right)$. First, $D^{\prime}$ defines the public parameter as $p p:=\left(G, H:=G, G_{T}\right.$, $N, g, e, m, n$, pars $\left.^{\prime}\right)$, for $m: h^{\prime} \mapsto e\left(g_{1}, h^{\prime}\right)$, pars $^{\prime}:=\left(g_{1}, g_{4}, g_{1}^{\mathbf{w}}, h:=g, h^{\mathbf{w}}\right)$, for $\mathbf{w} \leftarrow\left(\mathbb{Z}_{N}^{*}\right)^{n}$, and for some integer $n$ determined by $D^{\prime}$. Then, $D^{\prime}$ sends $\left(p p, g_{23} g_{4}^{\gamma}, g_{12}, \mathbf{T}, \mathbf{T}^{\mathbf{w}}\right)$, for $\gamma \leftarrow \mathbb{Z}_{N}^{*}$, to $D$. Eventually, $D$ outputs a value which is forwarded by $D^{\prime}$ to its own challenger.

Analysis. Note that $p p$ is distributed as defined in LS2. If $\mathbf{T}=g_{12}^{\prime}$, then $\left(g_{12}^{\prime},\left(g_{12}^{\prime}\right)^{\mathbf{w}}\right)$ is distributed as SampG $(p p) \cdot \widehat{\operatorname{SampG}}(p p, s p)$, for suitable $s p$, as needed and, hence, we have that $\operatorname{Pr}\left[D^{\prime}\left(\right.\right.$ pars, $\left.\left.g_{12}^{\prime}\right)=1\right]=$

$\operatorname{Pr}\left[D\left(p p, g_{23} g_{4}^{\gamma}, g_{12},\left(g_{12}^{\prime},\left(g_{12}^{\prime}\right)^{\mathbf{w}}\right)\right)=1\right]$ follows. Otherwise, if $\mathbf{T}=g_{13}^{\prime}$, then $\left(g_{13}^{\prime},\left(g_{13}^{\prime}\right)^{\mathbf{w}}\right)$ is distributed as SampG $(p p) \cdot \widetilde{\operatorname{SampG}}(p p, s p)$, for suitable $s p$, as desired and, hence, $\operatorname{Pr}\left[D^{\prime}\left(\right.\right.$ pars,$\left.\left.g_{13}^{\prime}\right)=1\right]=$

$\operatorname{Pr}\left[D\left(p p, g_{23} g_{4}^{\gamma}, g_{12},\left(g_{13}^{\prime},\left(g_{13}^{\prime}\right)^{\mathbf{w}}\right)\right)=1\right]$ holds. As a consequence, (11) follows.

Nested-hiding indistinguishability. We prove the following lemma

Lemma 11 (DS3 to NH). For any PPT adversary $D$ with running time $t$ on $N H$ of ENDSG $_{\mathrm{co}}$ there is a distinguisher $D^{\prime}$ on DS3 with running time $t^{\prime} \approx t$ such that

$$
\operatorname{Adv}_{\mathrm{ENDSG}_{\mathrm{co}}, \mathrm{G}, D}^{\mathrm{nh}}(k) \leq \operatorname{Adv}_{\mathrm{G}, D^{\prime}}^{\mathrm{ds} 3}(k),
$$

for $\mathrm{G}$ as defined above. Hence, NH holds under DS3.

Proof. The proof follows the same strategy as shown in Chen and Wee's work [11] except that we have to integrate two coprime-order semi-functional generators $\widehat{h}$ and $\widetilde{h}$ instead of just one as in [11]. 
Description. The challenge input to $D^{\prime}$ is provided as (pars, $\left.\mathbf{T}\right)$, where $\mathbf{T}:=(\widehat{\mathbf{T}}, \widetilde{\mathbf{T}})$ is either $\left(g_{2}^{x y}, g_{3}^{x y}\right)$ or $\left(g_{2}^{x y+\gamma^{\prime}}, g_{3}^{x y+\gamma^{\prime}}\right)$, for

$$
\text { pars }=:\left(G, G_{T}, N, e, g_{1}, g_{2}, g_{3}, g_{4}, g_{2}^{x} \widehat{X}_{4}, g_{2}^{y} \widehat{Y}_{4}, g_{3}^{x} \widetilde{X}_{4}, g_{3}^{y} \widetilde{Y}_{4}\right),
$$

for $\widehat{X}_{4}, \widehat{Y}_{4}, \widetilde{X}_{4}, \widetilde{Y}_{4} \stackrel{g}{\leftarrow} G_{p_{4}}, x, y \leftarrow \mathbb{Z}_{N}^{*}$, and for $\gamma^{\prime} \leftarrow \mathbb{Z}_{N}^{*}$. Furthermore, $D^{\prime}$ receives an auxiliary input $i \in\left[\left\lfloor\frac{n}{2}\right\rfloor\right]$, for some integer $n \in \mathbb{N}$ determined by $D^{\prime}$. First, $D^{\prime}$ samples $r, \hat{r}, \tilde{r}, \hat{s}, \tilde{s} \leftarrow \mathbb{Z}_{N}^{*}, \mathbf{R}_{4}^{\prime} \stackrel{g}{\leftarrow}\left(G_{p_{4}}\right)^{n}, \mathbf{w}^{\prime} \leftarrow\left(\mathbb{Z}_{N}^{*}\right)^{n}$, and sets

$$
\begin{array}{r}
h:=\left(g_{1} g_{2} g_{3} g_{4}\right)^{r}, \quad \widehat{h}:=\left(g_{2} g_{4}\right)^{\hat{r}}, \quad \widetilde{h}:=\left(g_{3} g_{4}\right)^{\tilde{r}}, \\
\widehat{\mathbf{g}}_{-(2 i-1)}:=\left(g_{2}^{\hat{s}}, g_{2}^{\hat{s} \mathbf{w}^{\prime}}\right)_{-(2 i-1)}, \quad \widetilde{\mathbf{g}}_{-2 i}:=\left(g_{3}^{\tilde{s}}, g_{3}^{\tilde{s} \mathbf{w}^{\prime}}\right)_{-(2 i)},
\end{array}
$$

where $h, \widehat{h}$, and $\widetilde{h}$ are generators of $G, G_{p_{2} p_{4}}$, and $G_{p_{3} p_{4}}$. Then, $D^{\prime}$ defines public parameter as

$$
p p:=\left(G, H:=G, G_{T}, N, g, e, n, m, \text { pars }^{\prime}\right),
$$

for $m: h^{\prime} \mapsto e\left(g_{1}, h^{\prime}\right)$ and

$$
\begin{aligned}
\operatorname{pars}^{\prime} & :=\left(g_{1}, g_{4}, g_{1}^{\mathbf{w}^{\prime}}, h, h^{\mathbf{w}^{\prime}}\left(g_{2}^{y} \widehat{Y}_{4}\right)^{r \mathbf{e}_{2 i-1}}\left(g_{3}^{y} \widetilde{Y}_{4}\right)^{r \mathbf{e}_{2 i}} \mathbf{R}_{4}^{\prime}\right) \\
& =\left(g_{1}, g_{4}, g_{1}^{\mathbf{w}}, h, h^{\mathbf{w}} \mathbf{R}_{4}\right),
\end{aligned}
$$

where $\mathbf{e}_{j}$ is the $j$-th unit vector of length $n$ and, implicitly, we have

$$
\mathbf{w}=\left\{\begin{array}{l}
\mathbf{w}^{\prime} \bmod p_{1} p_{4} \\
\mathbf{w}^{\prime}+y \cdot \mathbf{e}_{2 i-1} \bmod p_{2} \quad \text { and } \quad \mathbf{R}_{4}=\mathbf{R}_{4}^{\prime}+\widehat{Y}_{4}^{r} \cdot \mathbf{e}_{2 i-1}+\tilde{Y}_{4}^{r} \cdot \mathbf{e}_{2 i} . \\
\mathbf{w}^{\prime}+y \cdot \mathbf{e}_{2 i} \bmod p_{3}
\end{array}\right.
$$

Now, by running the algorithm from [12, Lemma 6] on input $\left(1^{q^{\prime}},\left(g_{2}, g_{4}, g_{2}^{x} \widehat{X}_{4}, g_{2}^{y} \widehat{Y}_{4}, \widehat{\mathbf{T}}\right)\right)$ and on input $\left(1^{q^{\prime}},\left(g_{3}, g_{4}, g_{3}^{x} \widetilde{X}_{4}, g_{3}^{y} \widetilde{Y}_{4}, \widetilde{\mathbf{T}}\right)\right), D^{\prime}$ generates tuples

$$
\left(g_{2}^{\hat{r}_{j}} \widehat{X}_{4, j}, \widehat{\mathbf{T}}_{j}\right)_{j=1}^{q^{\prime}} \text { and }\left(g_{3}^{\tilde{r}_{j}} \widetilde{X}_{4, j}, \widetilde{\mathbf{T}}_{j}\right)_{j=1}^{q^{\prime}},
$$

respectively, where

$$
\widehat{\mathbf{T}}_{j}= \begin{cases}g_{2}^{\hat{r}_{j} y} \cdot \widehat{Y}_{4, j}, & \text { if } \widehat{\mathbf{T}}=g_{2}^{x y} \\ g_{2}^{\hat{r}_{j} y} \cdot \widehat{Y}_{4, j} \cdot g_{2}^{\hat{\gamma}^{\prime}{ }_{j}}, & \text { if } \widehat{\mathbf{T}}=g_{2}^{x y+\gamma^{\prime}}\end{cases}
$$

and

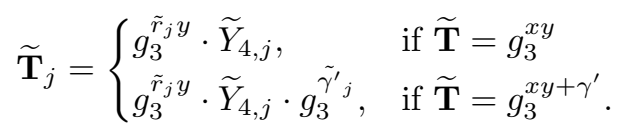

Further, $D^{\prime}$ samples $r_{j}^{\prime} \leftarrow \mathbb{Z}_{N}^{*}, \mathbf{X}_{4, j}^{\prime} \stackrel{g}{\leftarrow}\left(G_{p_{4}}\right)^{n}$, for all $j \in\left[q^{\prime}\right]$, and sends

$$
\left(p p, \widehat{h}, \widetilde{h}, \widehat{\mathbf{g}}_{2 i-1}, \widetilde{\mathbf{g}}_{2 i},\left(\mathbf{T}_{1}, \ldots, \mathbf{T}_{q^{\prime}}\right)\right)
$$


to $D$, where

$$
\begin{aligned}
\mathbf{T}_{j}= & \left(h^{r_{j}^{\prime}} \cdot g_{2}^{\hat{r}_{j}} \widehat{X}_{4, j} \cdot g_{3}^{\tilde{r}_{j}} \widetilde{X}_{4, j},\left(h^{r_{j}^{\prime}} \cdot g_{2}^{\hat{r}_{j}} \widehat{X}_{4, j} \cdot g_{3}^{\tilde{r}_{j}} \widetilde{X}_{4, j}\right)^{\mathbf{w}^{\prime}} \cdot\right. \\
& \left.\left(\left(g_{2}^{y} \widehat{Y}_{4}\right)^{r_{j}^{\prime} r} \widehat{\mathbf{T}}_{j}\right)^{\mathbf{e}_{2 i-1}} \cdot\left(\left(g_{3}^{y} \widetilde{Y}_{4}\right)^{r_{j}^{\prime} r} \widetilde{\mathbf{T}}_{j}\right)^{\mathbf{e}_{2 i}} \mathbf{X}_{4, j}^{\prime}\right) \\
= & \begin{array}{ll}
\left(h^{r_{j}}, h^{r_{j} \cdot \mathbf{w}} \cdot \mathbf{X}_{4, j}\right) & \text { if } \widehat{\mathbf{T}}_{j}=g_{2}^{\hat{r}_{j} y} \cdot \widehat{Y}_{4, j} \\
\left(h^{r_{j}}, h^{r_{j} \cdot \mathbf{w}} \cdot g_{2}^{\hat{\gamma}_{j} \mathbf{e}_{2 i-1}} \cdot g_{3}^{\tilde{\gamma}_{j} \mathbf{e}_{2 i}} \cdot \mathbf{X}_{4, j}\right) & \text { and } \widetilde{\mathbf{T}}_{j}=g_{3}^{\tilde{r}_{j} y} \cdot \widetilde{Y}_{4, j}=g_{2}^{\hat{r}_{j} y} \cdot \widehat{Y}_{4, j} \cdot g_{2}^{\hat{\gamma}_{j}} \\
& \text { and } \widetilde{\mathbf{T}}_{j}=g_{3} \tilde{r}_{j} y \cdot \widetilde{Y}_{4, j} \cdot g_{3}^{\tilde{\gamma}_{j}}
\end{array}
\end{aligned}
$$

for $h^{r_{j}}:=h^{r_{j}^{\prime}} \cdot g_{2}^{\hat{r}_{j}} \widehat{X}_{4, j} \cdot g_{3}^{\tilde{r}_{j}} \widetilde{X}_{4, j}$ and $\mathbf{X}_{4, j}:=\mathbf{X}_{4, j}^{\prime}+\widehat{Y}_{4}^{r_{j}^{\prime} r} \mathbf{e}_{2 i-1}+\widetilde{Y}_{4}^{r_{j}^{\prime} r} \mathbf{e}_{2 i}$ implicitly and $\mathbf{w}$ as above.

Analysis. Note that $p p$ is distributed as defined in NH. If $\mathbf{T}=\left(g_{2}^{x y}, g_{3}^{x y}\right)$, then $\widehat{\mathbf{T}}_{j}=g_{2}^{\hat{r}_{j} y} \cdot \widehat{Y}_{4, j}$ and $\widetilde{\mathbf{T}}_{j}=g_{3}^{\tilde{r}_{j} y} \cdot \widetilde{Y}_{4, j}$, for all $j \in\left[q^{\prime}\right]$, and, thus, $\left(\mathbf{T}_{1}, \ldots, \mathbf{T}_{q^{\prime}}\right)$ is distributed as $\left(\mathbf{h}_{1}, \ldots, \mathbf{h}_{q^{\prime}}\right)$, for suitable $s p$, as needed. Otherwise, if $\mathbf{T}=\left(g_{2}^{x y+\gamma^{\prime}}, g_{3}^{x y+\gamma^{\prime}}\right)$, then $\widehat{\mathbf{T}}_{j}=g_{2}^{\hat{r}_{j} y} \cdot \widehat{Y}_{4, j} \cdot g_{2}^{\hat{\gamma}_{j}}$ and $\widetilde{\mathbf{T}}_{j}=g_{3}^{\tilde{r}_{j} y} \cdot \widetilde{Y}_{4, j} \cdot g_{3}^{\tilde{\gamma}_{j}}$ for all $j \in\left[q^{\prime}\right]$, and, thus, $\left(\mathbf{T}_{1}, \ldots, \mathbf{T}_{q^{\prime}}\right)$ is distributed as $\left(\mathbf{h}^{\prime}{ }_{1}, \ldots, \mathbf{h}_{q^{\prime}}^{\prime}\right)$, for suitable $s p$, since $\left(\widehat{h}, g_{2}^{\hat{\gamma}_{j}} \cdot \widehat{Y}_{4, j}\right)$ and $\left(\widetilde{h}, g_{3}^{\tilde{\gamma}_{j}} \cdot \widetilde{Y}_{4, j}\right)$ are identically distributed as $\left(\widehat{h},(\widehat{h})^{\hat{\gamma}_{j}} \cdot \widehat{Y}_{4, j}\right)$ and $\left(\widetilde{h},(\widetilde{h})^{\tilde{\gamma}_{j}} \cdot \widetilde{Y}_{4, j}\right)$, respectively, for $\hat{\gamma}_{j}, \tilde{\gamma}_{j} \leftarrow \mathbb{Z}_{N}^{*}, \widehat{Y}_{4, j}, \widetilde{Y}_{4, j} \stackrel{g}{\leftarrow}$ $G_{p_{4}}$, for all $j \in\left[q^{\prime}\right]$.

Acknowledgments. We thank the anonymous reviewers for helpful remarks.

\section{References}

1. Abe, M., David, B., Kohlweiss, M., Nishimaki, R., Ohkubo, M.: Tagged one-time signatures: tight security and optimal tag size. In: Kurosawa, K., Hanaoka, G. (eds.) PKC 2013. LNCS, vol. 7778, pp. 312-331. Springer, Heidelberg (2013)

2. Bellare, M., Desai, A., Jokipii, E., Rogaway, P.: A concrete security treatment of symmetric encryption. In: 38th FOCS, pp. 394-403. IEEE Computer Society Press (October 1997)

3. Bellare, M., Desai, A., Pointcheval, D., Rogaway, P.: Relations among notions of security for public-key encryption schemes. In: Krawczyk, H. (ed.) CRYPTO 1998. LNCS, vol. 1462, pp. 26-45. Springer, Heidelberg (1998)

4. Bellare, M., Boldyreva, A., Micali, S.: Public-key encryption in a multi-user setting: security proofs and improvements. In: Preneel, B. (ed.) EUROCRYPT 2000. LNCS, vol. 1807, pp. 259-274. Springer, Heidelberg (2000)

5. Bellare, M., Waters, B., Yilek, S.: Identity-based encryption secure against selective opening attack. In: Ishai, Y. (ed.) TCC 2011. LNCS, vol. 6597, pp. 235-252. Springer, Heidelberg (2011)

6. Blazy, O., Kiltz, E., Pan, J.: (Hierarchical) identity-based encryption from affine message authentication. In: Garay, J.A., Gennaro, R. (eds.) CRYPTO 2014, Part I. LNCS, vol. 8616, pp. 408-425. Springer, Heidelberg (2014)

7. Boldyreva, A.: Strengthening security of RSA-OAEP. In: Fischlin, M. (ed.) CT-RSA 2009. LNCS, vol. 5473, pp. 399-413. Springer, Heidelberg (2009) 
8. Boneh, D., Boyen, X.: Short signatures without random oracles. In: Cachin, C., Camenisch, J.L. (eds.) EUROCRYPT 2004. LNCS, vol. 3027, pp. 56-73. Springer, Heidelberg (2004)

9. Boneh, D., Franklin, M.: Identity-based encryption from the weil pairing. In: Kilian, J. (ed.) CRYPTO 2001. LNCS, vol. 2139, pp. 213-229. Springer, Heidelberg (2001)

10. Cash, D.M., Kiltz, E., Shoup, V.: The twin diffie-hellman problem and applications. In: Smart, N.P. (ed.) EUROCRYPT 2008. LNCS, vol. 4965, pp. 127-145. Springer, Heidelberg (2008)

11. Chen, J., Wee, H.: Fully, (almost) tightly secure IBE and dual system groups. In: Canetti, R., Garay, J.A. (eds.) CRYPTO 2013, Part II. LNCS, vol. 8043, pp. 435-460. Springer, Heidelberg (2013)

12. Chen, J., Wee, H.: Dual system groups and its applications - compact hibe and more. Cryptology ePrint Archive, Report 2014/265 (2014). http://eprint.iacr.org/

13. Freeman, D.M.: Converting pairing-based cryptosystems from composite-order groups to prime-order groups. In: Gilbert, H. (ed.) EUROCRYPT 2010. LNCS, vol. 6110, pp. 44-61. Springer, Heidelberg (2010)

14. Galindo, D., Martín, S., Morillo, P., Villar, J.L.: Easy verifiable primitives and practical public key cryptosystems. In: Boyd, C., Mao, W. (eds.) ISC 2003. LNCS, vol. 2851, pp. 69-83. Springer, Heidelberg (2003)

15. Gentry, C.: Practical identity-based encryption without random oracles. In: Vaudenay, S. (ed.) EUROCRYPT 2006. LNCS, vol. 4004, pp. 445-464. Springer, Heidelberg (2006)

16. Gentry, C., Halevi, S.: Hierarchical identity based encryption with polynomially many levels. In: Reingold, O. (ed.) TCC 2009. LNCS, vol. 5444, pp. 437-456. Springer, Heidelberg (2009)

17. Groth, J., Sahai, A.: Efficient non-interactive proof systems for bilinear groups. In: Smart, N.P. (ed.) EUROCRYPT 2008. LNCS, vol. 4965, pp. 415-432. Springer, Heidelberg (2008)

18. Hofheinz, D., Jager, T.: Tightly secure signatures and public-key encryption. In: Safavi-Naini, R., Canetti, R. (eds.) CRYPTO 2012. LNCS, vol. 7417, pp. 590-607. Springer, Heidelberg (2012)

19. Lewko, A.: Tools for simulating features of composite order bilinear groups in the prime order setting. In: Pointcheval, D., Johansson, T. (eds.) EUROCRYPT 2012. LNCS, vol. 7237, pp. 318-335. Springer, Heidelberg (2012)

20. Libert, B., Joye, M., Yung, M., Peters, T.: Concise multi-challenge CCA-secure encryption and signatures with almost tight security. In: Sarkar, P., Iwata, T. (eds.) ASIACRYPT 2014, Part II. LNCS, vol. 8874, pp. 1-21. Springer, Heidelberg (2014)

21. Waters, B.: Dual system encryption: realizing fully secure IBE and HIBE under Simple assumptions. In: Halevi, S. (ed.) CRYPTO 2009. LNCS, vol. 5677, pp. 619-636. Springer, Heidelberg (2009)

22. Waters, B.: Efficient identity-based encryption without random oracles. In: Cramer, R. (ed.) EUROCRYPT 2005. LNCS, vol. 3494, pp. 114-127. Springer, Heidelberg (2005) 